\title{
Digitale Transformation und Human Resource Management
}

\section{Trends und Perspektiven einer zukunftsgerichteten Personalarbeit in Schweizer Großunternehmen}

\author{
Jochen Schellinger, Marlies Goedermans, Lars Patrick Kolb \\ und Yassin Sebai
}

\begin{abstract}
Zusammenfassung
Die voranschreitende digitale Transformation elementarer Unternehmensprozesse schließt auch die betrieblichen Querschnittsfunktionen mit ein. Dies gilt in besonderem Maße für das Human Resource Management von Unternehmen, das sowohl als Objekt als auch als ein Treiber dieser digitalen Veränderungen in Erscheinung treten kann. Strategisch relevante Veränderungen wie Cloud Computing, Big Data und Social Media Management sowie Mobility-Trends wirken sich mittelbar und unmittelbar auch auf die Gestaltungsbereiche betrieblicher Personalarbeit aus. Der vorliegende Beitrag setzt sich theoretisch und empirisch mit der Frage nach der Relevanz und Konsequenz der Digitalisierung für das Personalmanagement in Schweizer Großunternehmen auseinander. Im Mittelpunkt steht die Vorstellung der Ergebnisse einer qualitativen Befragung von sechzehn HR-Managern führender Schweizer Großunternehmen aus verschiedensten Wirtschaftsbereichen und eines weltweit marktführenden eHR-Softwareanbieters. Grundlage hierfür war die Entwicklung eines Reifegradmodells, das versucht, die Digitalisierung anhand von acht Dimensionen des digitalen Wandels
\end{abstract}

J. Schellinger $(\square) \cdot$ M. Goedermans $\cdot$ Y. Sebai

Bern, Schweiz

E-Mail: jochen.schellinger@bfh.ch

M. Goedermans

E-Mail: marlies.goedermans@sap.com

Y. Sebai

E-Mail: yaessu@msn.com

L. P. Kolb

Ittigen, Schweiz

E-Mail: lars.kolb@gmx.ch 
abgestuft zu spezifizieren. Die Schweizerische Unternehmenslandschaft scheint sich aufgrund der Erkenntnisse der Befragung in Bezug auf die Digitalisierung noch in einer Aufbauphase zu bewegen. Dies gilt auch hinsichtlich des Umsetzungsstands von Digital HRM-Ansätzen in den Unternehmen. Die Personalbereiche können und dürfen sich dem Digitalisierungstrend aber nicht verschließen und sollten künftig eine noch aktivere Rolle bei der Umsetzung von Digitalisierungsstrategien spielen.

\subsection{Einleitung}

Mit „Digitalisierung“ wird ein globaler Entwicklungstrend bezeichnet, der zunehmend alle Lebens- und Wirtschaftsbereiche durchdringt. Im ökonomischen Kontext steht Digitalisierung für die Vereinfachung und Effizienzsteigerung von Prozessen durch neue Möglichkeiten digitaler Technologien. Auch der betriebliche Personalbereich ist hiervon nicht ausgenommen. In welchem Ausmaß und in welcher Art und Weise die Digitalisierung Personalprozesse verändert, ist für das Human Resource Management (HRM) in Schweizer Unternehmen empirisch allerdings noch kaum untersucht. Diese Grundfragestellung kann anhand von fünf leitenden Forschungsfragen weiter konkretisiert werden:

- Welche unter dem Begriff der Digitalisierung subsumierbaren Trends sind für das HRM weshalb von Bedeutung?

- Was sind in personaler Hinsicht zentrale Chancen und Problemfelder der Digitalisierung?

- Welche Konsequenzen und Gestaltungsempfehlungen lassen sich aus den wichtigsten Trends für die einzelnen Politikfelder des HRM ableiten?

- Wie sind einzelne Digitalisierungstrends aktuell im HRM Schweizer Unternehmen eingegangen?

- Wie bzw. in welche Richtung entwickelt sich das HRM Schweizer Unternehmen im Kontext der Digitalisierung in den nächsten Jahren weiter?

Als systematisierender Ausgangspunkt für die Identifikation von Digitalisierungspotenzialen im HRM dienen die acht Personalprozesse nach Nicolai (2009) sowie die vier Querschnittsfunktionen des Personalmanagements nach Thom (2001). Dabei wird von einer wechselseitigen Beeinflussung von HR-Hauptprozessen und -Querschnittsfunktionen ausgegangen. Bei der theoretisch-konzeptionellen Aufbereitung wurden 120 einschlägige Quellen aus dem deutschen und angelsächsischen Sprachraum zur Fundierung der qualitativen Befragungen von 17 (e)HR-Fachexperten Schweizer Unternehmen analysiert und die Erkenntnisse anhand eines Reifegradmodells zur Digitalisierung in der Schweiz zusammengefasst, das im empirischen Teil der Untersuchung den Rahmen für eine praxeologische Prüfung und Interpretation absteckt. 


\subsection{Personalbezogene Aspekte der digitalen Transformation}

Startpunkt für die konzeptionelle Aufbereitung zur Beantwortung der Forschungsfragen ist eine Präzisierung relevanter Begrifflichkeiten und Rahmensetzungen aus der Human Resource Management-Literatur. Es folgen eine Konkretisierung des Digitalisierungskontexts besonders relevanter Ansätze und Einzelaspekte für das HRM sowie eine Marktbetrachtung für digitale HR-Produkte und -Services. Im Anschluss an eine Reflexion potenzieller Problemfelder wird das für die empirische Analyse strukturgebende Reifegradmodell abgeleitet.

\subsubsection{Begriffliche und konzeptionelle Grundlagen}

Wie nahezu für alle Managementbegrifflichkeiten gibt es auch für das HRM nicht eine Definition, sondern viele. Human Resource Management wird oft synonym mit den Begriffen Personalwirtschaft, Personalwesen, Personalmanagement oder Strategisches Personalmanagement verwendet (Karaus 2003, S. 8 f.). Es geht bei allen Begriffen aber stets um den betrieblichen Produktionsfaktor Mensch und dessen Leistungen sowie dessen richtungsweisende Führung (Kolb 2010, S. 5). Seit den 1990er-Jahren wird HRM als zunehmend bedeutsame Unternehmensfunktion gesehen, und die Mitarbeitenden werden als wertgenerierendes zentrales Kapital des Unternehmens betrachtet. Das HRM hat als betriebliche Funktion die Aufgabe, die richtigen Menschen zur richtigen Zeit in der richtigen Anzahl mit der adäquaten Qualifikation am richtigen Ort der Leistungserstellung in der Organisation einzusetzen (Haubrock 2004, S. 17 f.). Seit der Jahrtausendwende haben neue unternehmensinterne und -externe Herausforderungen das HRM geprägt: der wachsende Wettbewerbsdruck erfordert ein kontinuierliches Kosten- und Veränderungsmanagement; die Globalisierung forciert unternehmerisches Agieren und führt zu globalen Organisationen mit multinationalen Kulturen; die technologische Entwicklung erfordert neue Qualifikationen der Mitarbeitenden sowie deren Anpassungsfähigkeit; der demografische Wandel beeinflusst sehr stark die internen und externen Arbeitsmärkte und den Erwartungen der Mitarbeitenden in Bezug auf Arbeitszeitmodelle, Mitbestimmung am Arbeitsplatz oder Formen neuer Arbeitsorganisationen muss Rechnung getragen werden (Wertewandel). Hieraus resultieren neue Belegschafts- und Leistungsstrukturen (Selke 2012, S. 207 f.).

Eine Vielzahl der Unternehmen misst heute dem HRM einen hohen Stellenwert bei. Je höher die strategische Priorisierung des Funktionsfelds, desto weiter oben findet sich die Personalabteilung und das HR-Management in der Unternehmenshierarchie (Nicolai 2009, S. 20). Die hierarchische Aufhängung korrespondiert dabei oftmals mit der Unternehmensgröße (Heymann 2009, S. 26). Kleine und mittelständische Unternehmen (KMU) weisen der Personalabteilung in der Regel nicht eine höchste Priorität zu. Der Personalbereich ist bei mittleren Unternehmen im Normalfall erst auf der 
zweiten Hierarchieebene positioniert. Häufig wird die Personalabteilung dann einer Verwaltungsabteilung unterstellt oder ist in andere Abteilungen integriert. In Kleinunternehmen existiert aufgrund des limitierten Aufgabenumfangs in der Regel keine originäre Personalabteilung. Die Aufgaben werden meist von einer administrativen Verwaltungsstelle oder dem Eigentümer selbst ,nebenbei“ erledigt (Nicolai 2009, S. 20).

In Verbindung mit der Personalfunktion werden in Unternehmen in der Regel ökonomische Ziele (optimale Personalressourcenallokation und Steigerung der Arbeitsleistung) und soziale Ziele im Sinne einer Bedürfnisbefriedigung der Mitarbeitenden verfolgt. Das resultierende Spannungsverhältnis muss bei der Ausgestaltung der Personalstrategien und -prozesse vom HRM berücksichtigt werden (Scherm und Süss 2011, S. 6). Die zunehmende Komplexität und Verrechtlichung der Arbeitsbeziehungen macht diese Aufgabe nicht leichter.

Die Aufgabenstellungen des HRM können in Anlehnung an die Prozessperspektive von Nicolai (2009) in insgesamt acht Teilprozesse untergliedert werden: Personalbedarfsplanung, Personalbeschaffung, Personalauswahl, Personaleinführung und -einarbeitung, Personaleinsatz und -erhaltung, Personalbeurteilung sowie Personalentwicklung und -freisetzung. Bei der elementaren Personalbedarfsplanung geht es um die zukunftsgerichtete Bestimmung der notwendigen personellen Ressourcen zur Erreichung der Unternehmensziele. Ziel ist die kontinuierliche Deckung des quantitativen und qualitativen Bedarfs an Arbeitsleistung unter Beachtung der zeitlichen und örtlichen Notwendigkeit (Nicolai 2009, S. 31 ff.). Die Personalbeschaffung verfolgt das Ziel, die richtige Anzahl an Mitarbeitenden mit der passenden Qualifikation zum richtigen Zeitpunkt und für die richtige Zeitspanne am passenden Arbeitsort zu positionieren (a. a. O., S. 48 ff.). Sie kann intern oder extern erfolgen, wobei in Verbindung mit der Digitalisierung für die externe Personalbeschaffung das E-Recruiting in den letzten Jahren massiv an Bedeutung gewonnen hat. Bei der Personalauswahl wird das Ziel verfolgt, diejenigen Bewerber zu ermitteln, die mit ihren Qualifikationen und ihrer Persönlichkeit am geeignetsten zur Besetzung vakanter Stellen erscheinen. Fehlerhafte Auswahlentscheidungen sind in der Regel mit beträchtlichen Kosten verbunden (Scherm und Süss 2013, S. 54). Zur Personalauswahl stehen diverse Selektionsverfahren zur Verfügung, die üblicherweise kombiniert werden. Nach Arbeitseintritt dienen Einführungs- und Entwicklungsmaßnahmen einer systematischen Integration ausgewählter Personen in die Organisation (Nicolai 2009, S. 125). Personaleinsatz und Personalerhaltung stehen für sämtliche Aspekte des HRM, bei denen es darum geht, Mitarbeitende optimal zu beschäftigen, ihre Qualifikationen sowie ihr Potenzial für die Organisation zu erhalten, zu fördern und den Unternehmensbedürfnissen anzugleichen. Die Mitarbeitenden sollen zu Leistungssteigerungen motiviert und perspektivisch an das Unternehmen gebunden werden (Nicolai 2009, S. 133). Von besonderer Bedeutung sind hierbei adäquate Anreizsysteme zur materiellen und immateriellen Incentivierung. Die Personalbeurteilung dient als institutionalisiertes System und Verfahren, welches die Leistungsergebnisse, das Arbeits-, Führungs- und Sozialverhalten sowie das Potenzial des Mitarbeiters kontrolliert (Nicolai 2009, S. 236 ff.). Aktuell zeichnet sich in der 
Praxis ein Trend zur Durchführung von Mehrfachbeurteilungen ab (z. B. 360-Feedbacks). Die Personalentwicklung stellt gemäß Nicolai (2009, S. 266) eine ,immaterielle Investition ins Humankapital“ dar. Im Zentrum stehen die Vermittlung und der Erwerb von neuen Qualifikationen der Mitarbeitenden zur Nachwuchssicherung und zur Instandhaltung und Intensivierung des betrieblichen Wissens (Wachter 2015). In Verbindung mit der Digitalisierung kann vor allem auch auf die zunehmende Bedeutung von agilen E-Learning-Ansätzen verwiesen werden (z. B. Blended Learning/Distance Learning) (Kolb 2008, S. 473 f.). Unter Personalfreisetzung versteht man sämtliche Maßnahmen zur Dezimierung einer personellen Überdeckung in qualitativer und quantitativer sowie zeitlicher und örtlicher Perspektive (Nicolai 2009, S. 326 f.). Häufigste Beweggründe zur Personalfreisetzung sind in der Praxis Sanierungen, Reorganisationen und Standortschließungen (Stock-Homburg 2008, S. 216 f.). Die sorgfältige Durchführung des Freistellungsprozesses hat einen beträchtlichen Einfluss auf das Unternehmensimage und führt zu entsprechenden Wettbewerbsvorteilen oder -nachteilen bei der künftigen Personalbeschaffung auf dem Arbeitsmarkt.

Die angeführten Personalprozesse stehen in enger Wechselwirkung mit den personalen Querschnittsfunktionen zur Erschließung von im HR-Bereich verankerten Erfolgspotenzialen (Thom 2001). Dem Personalcontrolling kommt die Aufgabe zu, Planungs-, Steuerungs- und Kontrollfunktionen zu unterstützen und wahrzunehmen sowie die personale Informationsversorgung sicherzustellen (Thom 2001, S. 125 f.). Ein systematisches Personalcontrolling kommt zumeist im Einsatz von Personalkennzahlensystemen zum Ausdruck. Die Digitalisierung dürfte in dem Zusammenhang zu völlig neuen integrierten Analyse- und Steuerungsmöglichkeiten beitragen. Das Personalmarketing setzt sich vorrangig mit dem Employer Branding und der hiermit einhergehenden Positionierung als attraktiver Arbeitgeber auf dem Arbeitsmarkt auseinander (Thom 2001, S. 126 f.). Die Digitalisierung hat auch hier eine zunehmende Fülle neuer zielgruppenorientierter Vermarktungsoptionen hervorgebracht (z. B. Social Media-HR-Marketing) (Beck 2008, S. 49). Im Rahmen der Personalinformation ist es Aufgabe des HRM, eine verständnisfördernde innerbetriebliche Kommunikation (Cantin 1999) zu realisieren. Eine jüngere Untersuchung bei den 500 umsatzstärksten Unternehmen Deutschlands (Spachmann und Huck-Sandhu 2013, 2015) zeigt in Verbindung mit der Digitalisierung die Tendenz auf, dass sich die Personalinformation zunehmend weg von einer einseitigen Sprachrohrfunktion der Geschäftsleitung hin zu verselbstständigten dialogorientierten Austauschplattformen verschiebt, die neue Kollaborationsmöglichkeiten eröffnen und zu einer offenen Kommunikationskultur beitragen (Huck-Sandhu 2015, S. 7 f.). Die Organisation des Personalmanagements schließlich befasst sich mit der Regelung des Zusammenspiels der involvierten Aufgabenträger innerhalb des Personalbereichs und zwischen HRM und Linienführungkräften (Arbeitsteilung und Koordination) (Thom 2001, S. 127 f.).

Das strategische Personalmanagement ist als potenzialorientierte Metafunktion über den Personalprozessen und den HR-Querschnittsfunktionen positioniert und befasst sich mit grundlegenden Gestaltungsoptionen zur personalseitigen Implementierung der 


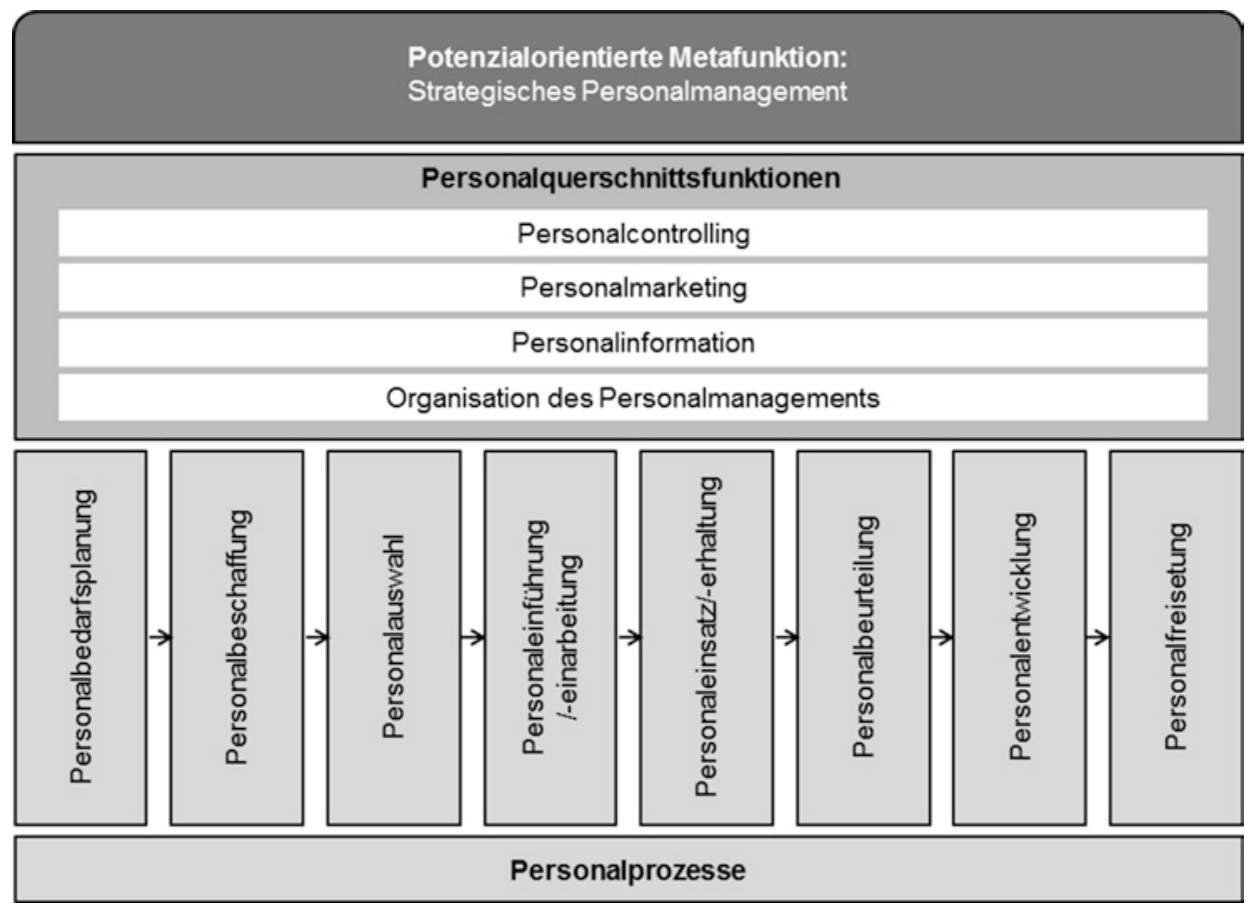

Abb. 8.1 Handlungs- und Gestaltungsbereiche des HRM. (Eigene Darstellung in Anlehnung an Nicolai 2009, S. 5 f.; Thom 2001, S. 118)

Unternehmensstrategie. Die drei Handlungs- und Gestaltungsbereiche des HRM sind gesamthaft in Abb. 8.1 dargestellt und dienen als erster Bezugsrahmen für die Analyse der HR-bezogenen Digitalisierungsaspekte.

\subsubsection{Human Resource Management im Digitalisierungskontext}

Die Digitalisierung kann als Metatrend verstanden werden, der eine Veränderung der Arbeitswelt, der Unternehmens- und Wissenskultur sowie der damit korrespondierenden Führungsverständnisse nach sich zieht (Cachelin 2013a). Westermann et al. (2014) sehen die digitale Transformation als technikbasierte Performance- oder Reichweitenerhöhung von Unternehmen, die mit einer Transformation von Betriebsprozessen, Kundenerlebnissen und der Geschäftsmodelle verbunden ist. Digitalisierung steht ferner für eine zunehmende Immaterialisierung von Produkten, Prozessen und Ressourcen von Unternehmen (Cachelin 2013a) und geht mit einer Mediatisierung, einer rapiden Beschleunigung der Arbeitsabläufe sowie einer wachsenden Verdichtung und Komplexitätssteigerung der Tätigkeiten einher (Bankl 2014, S. 306). 
In Bezug auf das HRM gibt es eine Fülle von Verbindungslinien zum Digitalisierungskontext. Eine eindeutige Festlegung dessen, was Digitalisierung für das HRM bedeutet, gibt es derzeit noch nicht. Hackl und Gerpott (2014) sehen als Folge der Digitalisierung eine Verschmelzung von physikalischer und virtueller Arbeitswelt, die sich auf das HRM nachhaltig auswirken wird. Eine zunehmende Virtualisierung der Arbeit und Interaktionsbedarf mit intelligenten Maschinen, erhöhte Mitarbeitermobilität und neue personale Anforderungsprofile führen zu Neuerungen vor allem hinsichtlich der Auswahl und Entwicklung von Arbeitskräften mit den künftig benötigten technologischen Kompetenzen sowie in Bezug auf die Neugestaltung von Arbeits- und Geschäftsmodellen. Die Digitalisierung geht einher mit einer resultatsorientierten Auf- und Ablauforganisation der Personalprozesse (Gora 2016) bei der administrative Routinearbeiten automatisiert werden. Die Vernetzung der HRM-Instrumente erlaubt das Einbringen fundierterer und neuer HR-Informationen in den strategischen Entwicklungsprozess. So geht aus dem Ergebnisbericht der HR-Trendstudie 2015 von Kienbaum (2015) hervor, dass rund $70 \%$ aller Studienteilnehmenden in Data Analytics ein hochgradiges Nutzungspotenzial sehen. In diesem Zusammenhang wird der Einsatz von künstlicher Intelligenz im HRM spürbar zunehmen und Tools wie Social Media, Mobile Applications und Cloud-Lösungen werden künftig zum HRM-Standard gehören. In Bezug auf die zu entwickelnden Führungskonzepte werden Fähigkeiten zum Change Management sowie zur Vernetzung und wertschätzenden Kommunikation noch wichtiger. Insgesamt gewinnt das HRM durch die Digitalisierung wohl an Bedeutung (Holtbrügge 2015, S. 5).

Es gibt zwar eine ständig wachsende Zahl an theoretisch-konzeptionellen Beiträgen, literarischen Werken und empirischen Studien, die das Ziel verfolgen, in den Umgang mit der digitalen Transformation auf der Metaebene einzuführen und Entwicklungspotenziale und geschaffene Herausforderungen für eine Volkswirtschaft, Branche oder Unternehmung aufzuzeigen. Die wenigsten Arbeiten beschäftigen sich jedoch explizit mit der Digitalisierung im Kontext HRM. Gemäß einer Studie von Ernst \& Young (2016) bei 700 mittelständischen Schweizer Firmen mit 30 bis 2000 Mitarbeitenden kann aus der Unternehmenspraxis heraus festgehalten werden, dass mehr als die Hälfte der untersuchten Fälle den digitalen Technologien nur eine marginale Bedeutung zuweisen. Jedoch muss angemerkt werden, dass in der Schweiz offenbar ein „digitaler Graben“ zwischen Großkonzernen und KMU besteht. Während Großunternehmen sich seit geraumer Zeit mit den Chancen und Herausforderungen der Digitalisierung beschäftigen, hinken kleinere und mittlere Betriebe hinterher. Eine in jüngerer Zeit durchgeführte Studie der Hochschule für Wirtschaft Zürich (HWZ) mit 463 befragten Personen mit und ohne Kaderfunktion in Schweizer Unternehmen stellte entsprechend fest, dass über $50 \%$ der Unternehmen als ,digitale Dinosaurier“ kategorisiert werden können. Insgesamt scheint in der Schweizer Wirtschaft nach wie vor digitales Know-how sowohl auf Führungs- als auch auf Mitarbeiterebene zu fehlen (HWZ 2015). Der Digital Transformation Report von Berghaus et al. (2015) hat versucht, per Online-Umfrage mit 196 Teilnehmenden, von denen $83 \%$ aus der Schweiz stammen, die digitalen Fähigkeiten 
der befragten Unternehmen zu ermitteln. Durch die Gewinnung der Daten konnte ein Digital Maturity Model entwickelt werden, das neun relevante Fähigkeiten für die digitale Transformation zusammenfasst. Die digitale Reife einer Unternehmung konnte anhand von fünf Reifegraden aufgezeigt werden. Berghaus et al. (2015, S. 7) betonen in dem Zusammenhang, dass es für eine erfolgversprechende digitale Transformation entscheidend sei, dass die Geschäftsleitung eine unterstützende Rolle einnehme, das mittlere Management zum Handeln aufgefordert werde, die Transformation eine entsprechende Strategie benötige, der Spirit einer digitalen Unternehmung spürbar sein sowie aus einzelnen Projekten ein Programm gemacht werden müsse.

Forschungsseitig ist die Thematik Digitalisierung und HRM in der Schweiz im Vergleich zu Deutschland, Österreich und vor allem den USA noch relativ wenig erschlossen. Cachelin veröffentlicht seit 2012 in regelmäßigen Abständen wissenschaftliche Beiträge zur Bedeutung des technologischen Wandels und der resultierenden Relevanz neuer technologischer Tools für die Personalabteilungen. Er sieht die Digitalisierung als Auslöser eines umfassenden Change-Prozesses (Cachelin 2014a). Die Metauntersuchung „HRM-Trendstudie 2013“ (Cachelin 2014b) analysiert zehn Studien, die sich mit der Zukunft der Personalarbeit befassen. Sie fokussiert auf die Darstellung der benötigten wertschaffenden HR-Kompetenzen in einer digitalen Welt: die Schaffung eines Digitalisierungsverständnisses, die Nutzung von digitalen HR-Instrumenten zur verstärkten Wirkung des HRM, das Erlangen der Datenkompetenz zur Erarbeitung einer Datenstrategie und eines effektiven Controllings sowie die Positionierung des HR-Bereichs innerhalb des Unternehmens durch das richtige Angebot und konsequentes Marketing. Die Folgestudie zu den Risiken eines digitalen Arbeitsumfelds mit 232 Teilnehmenden aus Deutschland, Österreich und der Schweiz zeigt auf, dass eine Annäherung von HR- und IT-Abteilungen für ein integriertes Risiko- und Innovationsmanagement erforderlich scheint (Cachelin 2015). Die HRM-Trendstudie von Kienbaum (2015) basiert auf einer Befragung von 187 Personalverantwortlichen führender Unternehmen im deutschsprachigen Raum (Anteil Deutschland 86\%, Schweiz $2 \%$ ). Sie zeigt erste Trends in der Nutzung von Digitalisierungs-Tools im HRM auf und räumt der digitalen Transformation ein hohes Erfolgspotenzial ein. Die Befragten sehen derzeit noch einen geringen Nutzungsgrad von Data Analytics, künstlicher Intelligenz, Social Media, Mobile Applications und Cloud-Lösungen im HRM, gehen aber von einem großen Potenzial für das HRM der Zukunft aus. Auf die wichtige Bedeutung eines adäquaten Umgangs mit dem Thema Datenschutz im Kontext der Digitalisierung verweist die Deutschlandstudie von Olsok et al. (2016). Die umfassende Befragung von 532 Führungskräften im deutschsprachigen Raum (Anteil Schweiz $32 \%$ ) durch Eilers et al. (2016) kommt zum Schluss, dass die digitale Transformation in Unternehmen nur dann gelingen kann, wenn dabei verstärkt auch kulturelle und soziale Aspekte einbezogen werden. Die jüngst erfolgte breitere Befragung von 480 HRM-Fachexperten (75\% aus Schweizer Unternehmen) identifiziert vor allem bei der Digitalisierung und im faktenbasierten HRM zukünftige Investitionsbedarfe (Winkler 2016). 


\subsubsection{Digitalisierungsansätze mit besonderer HRM-Relevanz}

Insgesamt scheinen vor allem vier technologische Entwicklungslinien der Digitalisierung für das HRM von besonderer Relevanz zu sein: Social Media, Data Mining, Cloud Computing und Mobility.

\section{Social Media}

In der digitalisierten Welt hat sich das Internet vom Informations- zum Mitmachmedium verändert, in dem das, was man von sich aktiv auf den Social-Media-Plattformen preisgibt, entscheidend dafür ist, wie man von anderen Personen und Unternehmen wahrgenommen wird (Schwarb und Vecchia 2016, S. 50). Folgende Eckzahlen mit Stand Anfang 2016 belegen eindrücklich die heutige Allgegenwart der sozialen Medien (Schwarb und Vecchia 2016, S. 52):

- 3,42 Mrd. Menschen sind aktiv im Internet (=46\% der Weltbevölkerung).

- $31 \%$ der Internetuser nutzen soziale Medien wie Facebook, Twitter etc.

- 1,5 Mrd. Menschen haben einen Facebook-Account.

- 3,5 Mio. Facebook-User gibt es in Österreich.

- 28 Mio. Facebook-User gibt es in Deutschland.

- 3,7 Mio. Facebook-User gibt es in der Schweiz.

- 3,1 Mio. Menschen sind mit mobilen Geräten in der Schweiz auf sozialen Medien aktiv.

Gerade junge Menschen diskutieren in den sozialen Medien wie Facebook, Xing oder LinkedIn über ihre Arbeitgeber, geben sich gegenseitig Tipps zur Bewerbung oder rekrutieren sogar Freunde als zukünftige Mitarbeiter (Cole 2015, S. 172). Das heutige Web 2.0 ermöglicht es außerdem, interessante Stellenangebote mit Freunden zu teilen oder Interessierten aus dem Bekanntenkreis direkt Links zu senden. So bieten zum Beispiel „Buddybroker“ die Möglichkeit, Freunden konkrete Jobangebote zu empfehlen (PwC 2013, S. 8). Deshalb nimmt die Bedeutung der Mitarbeitenden als Markenbotschafter eines Unternehmens deutlich zu. Die Digitalisierung ist außerdem mit einem zunehmenden Maß an Transparenz am Arbeitsmarkt verbunden. Ein Beleg hierfür sind etwa Arbeitgeberbewertungsplattformen wie Kununu (Jäger und Petry 2012, S. 21). Unternehmen müssen sich heute also im Rahmen eines „Employer Branding“ auch im Internet und in den sozialen Medien präsentieren und inszenieren (Cole 2015, S. 174). Die Auswahl relevanter Plattformen für in der Regel sehr zeitintensive Social Media-Aktivitäten hängt meist von der anzusprechenden Zielgruppe ab. Plattformen mit direkt steuerbaren, d. h. selbst erstellten Inhalten sind dabei von Plattformen mit nicht direkt steuerbaren, d. h. von Internetnutzern erstellten Inhalten abzugrenzen. Letztere werden von den Internetusern als vertrauenswürdiger eingestuft werden (Schwarb und Vecchia 2016, S. 57). Ein Überblick über die bekanntesten steuerbaren und nicht steuerbaren Social-Media-Plattformen und zugehörige HRM-Handlungsfelder gibt Tab. 8.1. 
Tab. 8.1 Steuerbare und nichtsteuerbare Social Media-Plattformen und HRM-Handlungsfelder. (Eigene Darstellung in Anlehnung an Schwarb und Vechia 2016, S. 71)

\begin{tabular}{l|l|l|l|l|l}
\hline \multicolumn{2}{|c|}{} & \multicolumn{2}{l}{ Relevante HRM-Handlungsfelder } \\
\hline Plattformen & $\begin{array}{l}\text { Direkte } \\
\text { Steuerbarkeit }\end{array}$ & $\begin{array}{l}\text { Employer } \\
\text { Branding }\end{array}$ & Stellenan-zeigen & $\begin{array}{l}\text { Bewerber- } \\
\text { recherche }\end{array}$ & $\begin{array}{l}\text { Active } \\
\text { Sourcing }\end{array}$ \\
\hline 1) Kununu & Nein & Ja & Ja & Nein & Nein \\
\hline 2) Glassdoor & Nein & Ja & Ja & Nein & Nein \\
\hline $\begin{array}{l}\text { 3) Staufenbiel } \\
\text { (Stellenbör- } \\
\text { senforum) }\end{array}$ & Nein & Nein & Ja & Nein & Nein \\
\hline 4) Xing & Ja & Ja & Ja & Ja & Ja \\
\hline 5) LinkedIn & Ja & Ja & Ja & Ja & Ja \\
\hline 6) Google+ & Ja & Ja & Ja & Ja & Ja \\
\hline 7) Facebook & Ja & Ja & Ja & Nein & Indirekt \\
\hline 8) Instagram & Ja & Ja & Ja & Nein & Nein \\
\hline 9) Snapchat & Ja & Bedingt & Bedingt & Nein & Nein \\
\hline 10) Twitter & Ja & Bedingt & Ja & Nein & Nein \\
\hline 11) WhatsApp & Ja & Bedingt & Bedingt & Nein & Nein \\
\hline
\end{tabular}

Bewertungsplattformen: 1) - 3)/Business-Plattformen: 4) - 5)/Private Netzwerke: 6) - 11)

Die Nutzung von Social Media im HRM steckt jedoch immer noch in den Kinderschuhen: Auch wenn in einer jüngeren Studie immerhin zwei Drittel der befragten 100 Unternehmen im Social Media-Bereich aktiv sind, so war bei lediglich knapp $20 \%$ Social Media ein etablierter Bestandteil des HRM (PwC 2013). Ein Social Media-Einsatz erfolgt überwiegend in der erfolgstransparenten Frühphase des Mitarbeitendenzyklus bei der Rekrutierung (91\%) sowie im Employer Branding (89\%). Ferner scheinen der Kontakthaltung zu ehemaligen Mitarbeitenden (29\%) und der Mitarbeitendenbindung (21\%) eine gewisse Bedeutung zuzukommen (PwC 2013, S. 15). Die vier von den Unternehmen am häufigsten genutzten Plattformen stehen mit Facebook (59\%), Xing (52\%), Linkedin (52\%) und YouTube (44\%) auch für die bekanntesten und etabliertesten Betreiber (PwC 2013, S. 12).

\section{Data Mining (Big Data/Business Intelligence)}

Unser Alltag wird mit der Zeit immer digitaler, was mit einer zunehmenden Datenproduktion einhergeht. Wir hinterlassen im Internet mit jedem Klick eine Spur, sei es beim Buchen der Ferien, beim Lesen eines Zeitungsartikels, beim Posten auf Facebook oder beim Bestellen eines Kochbuches. Gleiches gilt auch für die Arbeit: Jedes geführte Telefonat, jedes verschickte Mail, jedes abgespeicherte Dokument und jede besuchte Internetseite ist Teil der beruflichen Datenspur. Der Begriff „Big Data“, bzw. „Data Mining“ steht synonym für diese digitalisierungsbedingten riesigen Datensätze und deren Verwendung (Cachelin 2013c). 
Mit Big Data verbunden ist die Umwandlung des „Rohstoffs“ Informationen in verwertbares Wissen. Die bei der Big-Data-Verwertung eingesetzten Technologien und Methoden dienen dazu, Korrelationen und Muster zu erkennen, wo Menschen nur Datenchaos sehen (Cole 2015, S. 35). Es geht also um die Herstellung von „Transparenz“, die jedoch nicht immer willkommen ist. Gemäß der HR-Trendstudie 2013 denken $78 \%$ der Befragten, dass Big Data zu einem stärkeren Überwachen der Mitarbeitenden führt. Die Angst davor, dass der Datenschutz des eigenen Unternehmens nicht eingehalten wird, ist hoch. Lediglich $38 \%$ der Befragten wissen Bescheid, was ihr Unternehmen mit HR-Daten anstellt. Es besteht also ein großer Erklärungsbedarf hinsichtlich eines eventuellen HR Data Mining (Cachelin 2013b, S. 29).

Im HRM können Big Data-Analysen (Business Intelligence) dazu beitragen, Verbesserungspotenzialen auf die Spur zu kommen, sei es zum Beispiel bei der Identifikation von unterstützungsbedürftigen Mitarbeitenden oder bei der Effizienzsteigerung von Personalentscheidungen und -prozessen. Entsprechende HRM-Datenanalysen heben in der Regel auf die Beantwortung folgender Fragestellungen ab (Wagner AG 2016):

- Was ist passiert? (Beschreibung)

- Warum ist es passiert? (Analyse)

- Was wird passieren? (Voraussage)

- Was sollte geschehen? (Beeinflussung).

Große Anbieter wie SAP, Oracle und Microsoft bieten zur Beantwortung dieser Fragen dabei für Großunternehmen aber auch für KMU relativ kostengünstige Analytics-Lösungen an. Das Analytics-Tool von Microsoft etwa ist so konzipiert, dass auch Anwender ohne großes IT-Know-how einfach und schnell auf Personaldaten zugreifen, selbst Berichte erstellen und mit anderen Mitarbeitenden teilen können. Personalverantwortliche können Daten schnell und ohne Mühe visualisieren. Mit dem Analytics Tool sind Fluktuationen, demografische Entwicklungen, Abwesenheiten, Arbeitseintritte oder Lohnsummen analysierbar, visuell darstellbar und können für strategische Personalentscheidungen differenziert genutzt werden (Pentaho 2012, S. 1). Außerdem sind die Auswertungen auch über eine Mobile App auf dem Smartphone, Tablet oder Notebook jederzeit abrufbar.

\section{Cloud Computing}

„Cloud Computing“ steht für die Ausführung von Softwareprogrammen, die nicht auf lokalen Rechnern installiert sind, sondern von externen Rechnern, bzw. über das Internet aufgerufen werden. Im digitalen Zeitalter stellt die Verbindungsgeschwindigkeit über das Internet in der Regel kein Problem mehr dar, sodass immer mehr Dienste in diese „Software-Wolke“ verlagert werden. Anwendungen und der Speicherplatz für die Daten werden von einem externen Dienstleister mit Server-Farmen über das Internet bezogen, sodass man im Prinzip jederzeit und überall auf die Daten zugreifen kann (Focus 2010). Eine Mitarbeiterin kann so beispielswiese auf ihrem Tablet Dokumente erstellen und 
diese später auf dem Laptop abrufen und weiterbearbeiten. Es existieren unterschiedliche Arten von Cloud Computing: Infrastructure as a Service (IaaS - Miete von IT-Infrastruktur plus Wartung), Platform as a Service (PaaS - Bereitstellung von Entwicklungsumgebungen als Frameworks für selbst erstellte Programme) und Software as a Service (SaaS - Nutzung browserbasierter Standardsoftware via Internetbrowser) (Tetzel 2016; Botfrei Blog 2012).

Eine PwC-Studie (PwC 2014), an der sich knapp 270 in den USA ansässige Unternehmen beteiligten, veranschaulicht die Auslagerung von HR-Applikationen in die Cloud. Die Nutzung von Cloud-Lösungen unterschied sich demnach in Abhängigkeit von der Unternehmensgröße. So lagerten $70 \%$ der befragten Unternehmen mit weniger als 5000 Mitarbeitenden ihre HR-Applikationen in eine Cloud aus, aber nur $10 \%$ der Unternehmen mit mehr als 10.000 Mitarbeitenden. Mit zunehmender Kleinheit von Unternehmen konnte eine Tendenz zur HR-Cloud-Auslagerung ausgemacht werden. Die wichtigsten Gründe waren die Innovationsförderung, die Erhöhung der Flexibilität und eine Kostenreduzierung. Man konnte außerdem feststellen, dass Unternehmen welche HR-Anwendungen aus der Cloud einsetzen, mehr mobile Technologien einsetzen, deren Gebrauch ebenfalls deutlich zugenommen hat (2014 ca. $68 \%$ gegenüber $30 \%$ in 2013).

\section{Mobility}

Im Zeitalter der digitalen Vernetzung kommunizieren viele Mitarbeitende zunehmend auch von Zuhause aus mit Arbeitskollegen, Kunden, Lieferanten usw. Es ist für immer mehr Arbeitnehmende egal, wo sie arbeiten, da sie überall gleichermaßen gut erreichbar sind. Vor diesem Hintergrund verwenden Büroplaner und Architekten schon heute in wachsendem Maße die Formel $70 \mathrm{zu}$ 30: $70 \%$ der Mitarbeitenden befinden sich normalerweise an ihrem Schreibtisch im Unternehmen und $30 \%$ sind woanders. Dies geht einher mit der immer häufigeren Möglichkeit von Homeoffice-Tagen (Cole 2015, S. 178 f.). Eine Reihe von Unternehmen sieht diese Entwicklung aber durchaus auch kritisch. Sie befürchten einen Kontrollverslust. Die Digitalisierung fördert aber zwar eine Reduzierung der Arbeitskontrolle, erlaubt jedoch eine Beibehaltung der primär erfolgsrelevanten und unverzichtbaren Zielkontrolle (Jäger und Petry 2012, S. 127). Flexibilität wird von Kritikern ferner mit sinkender Produktivität in Verbindung gebracht. Letzteres ist aber heute wohl weniger eine Frage der Örtlichkeit als eine Frage der Unternehmenskultur. In dem Zusammenhang kann Homeoffice sogar dazu führen, dass Mitarbeitende effizienter arbeiten, denn sie sind motivierter und müssen Aufgaben verstärkt wertorientiert priorisieren.

\subsubsection{Demografie und Neupositionierung des HRM}

Zwei zentrale Aspekte für Digitalisierungsprozesse im HRM sind der demografische Wandel in der Schweizer Bevölkerung und substanzielle Veränderungen von Aufgabenstellung und Positionierung der Personalbereiche in Unternehmen. 


\section{Demografischer Wandel}

Seit Jahrzehnten konstant niedrige Geburtenraten und eine immer weiter steigende Lebenserwartung haben die Strukturen der Schweizer Bevölkerung stark verändert. Der Anteil der älteren Menschen hat stark zugenommen. Auf der anderen Seite ist der Anteil jüngerer Menschen erheblich gesunken. Damit verbunden ist ein wachsender Mangel an Fachkräften für die Wirtschaft. So sind in der Schweiz $41 \%$ (Deutschland $46 \%$ ) der Unternehmen bereits heute vom Fachkräftemangel betroffen. Unternehmen haben es schwer, passende Mitarbeiter zu rekrutieren. Im Jahr 2015 hat der Fachkräftemangel in der Schweiz um $8 \%$ im Vergleich zum Vorjahr zugenommen, was die Entwicklungsdynamik verdeutlicht (Tagesanzeiger 2015). Deutlich weniger kritisch als in der Schweiz ist diese Entwicklung etwa in Großbritannien und den Niederlanden, wo nur $14 \%$ der Firmen Schwierigkeiten haben, offene Stellen zu besetzen. Eine Reihe Schweizer Unternehmen muss immer wieder Kundenaufträge ablehnen, weil die nötigen Fachkräfte fehlen (Cole 2015, S. 38). Der demografische Wandel unterstützt also die Tendenz, dass junge Arbeitskräfte sich ihren Arbeitgeber aussuchen können, was die Unternehmen dazu zwingt, Employer Branding auch auf digitalen Kanälen zu betreiben, die durch potenzielle Fachkräfte privat genutzt werden (z. B. Social-Media-Plattformen).

\section{Veränderung des HRM-Aufgabenfelds durch den technologischen Wandel}

Die Personalabteilungen beschäftigen sich heute immer noch mehrheitlich mit traditionellen Aufgaben der Administration, Stammdatenpflege oder Rekrutierungsprozessen (Cachelin 2014b, S. 22). Zunehmend werden diese Aufgaben aber durch den Einsatz von Personalinformations- und -verwaltungssystemen obsolet. Eine wichtige Rolle spielen dabei Self Service-Lösungen für die Mitarbeitenden, beispielsweise bei der Spesenerfassung. IT-bedingte Aufgabenverschiebungen und Effizienzsteigerungen stellen in Verbindung mit der zunehmenden Bedeutung des Produktionsfaktors Personal im positiven Sinne eine Chance zur Neupositionierung des HRM dar. Die Trendstudie von Cachelin (2013b, S. 16) unterstützt diese Vermutung und identifiziert die Notwendigkeit zur Bildung neuer HRM-Geschäftsmodelle. Im Kontext des Digitalisierungstrends scheinen für die künftige Neuausrichtung von HR-Abteilungen insbesondere folgende Aufgabenstellungen prioritär (absteigende Wichtigkeit) (Cachelin 2013b, S. 15) (Reihenfolge absteigend):

- Talentmanagement

- Strategische Personalplanung

- HR Big-Data-Management

- HR IT-Management

- Führungskräfteentwicklung

- Mitarbeiterengagement.

Im Vordergrund steht zukünftig eine stärkere Wertschöpfung des HRM und deren Transparentmachung. Um dies zu erreichen, muss das HRM stärker über agile Strukturen in die Geschäftsprozesse des Unternehmens eingebunden werden (ebd.). 


\subsubsection{Anbieter und Nachfrager von HRM-Digitalisierungslösungen in der Schweiz}

Die derzeit besonders relevanten Anbieter von HRM-Softwarelösungen in der Schweiz werden in Anlehnung an den HR-Software-Report 2013 (HRM.ch 2013) unter Einbindung von Angaben der Firmenwebsites kurz vorgestellt. Der HR-Software-Report basiert auf einer Befragung von Personalexperten aus 138 Unternehmen in Deutschland, Österreich und der Schweiz. Die alphabetisch angeführten Unternehmen sind durchgängig mit einem Firmensitz in der Schweiz vertreten.

Das Schweizer Softwareunternehmen ABACUS beschäftigt 270 Mitarbeitende. Mit mehr als 100.000 verkauften Softwaremodulen und über 41.000 Kunden ist ABACUS der größte unabhängige Schweizer Anbieter von Business Software und konzentriert sich auf kleine und mittlere Unternehmen (KMU). Das Unternehmen ABACUS bietet zwei Hauptmodule für das HRM an: die Lohnbuchhaltung und ein HR-Gesamtpaket, das das Gros der Personalprozesse abdeckt. Schweizer Referenz-KMU sind u. a. die Herzog Haustechnik AG oder die Dr. Vollenweider AG Geotechnik.

Das amerikanische Unternehmen ADP ist seit über 60 Jahren weltweiter Führer im Bereich Gehalts- und HR-Management. Das in 135 Ländern aktive Unternehmen hat weltweit 637.000 Kunden. Das Unternehmen ADP ist in der Schweiz seit 30 Jahren vertreten und bedient über 250 Kunden in allen Geschäftsbereichen. Das Softwareangebot fokussiert auf Personalbeschaffung, -auswahl, -einsatz und -beurteilung. Ein bekannter Schweizer Referenzkunde ist die Syngenta AG.

Die deutsche Firma ATOSS ist über 25 Jahre am Markt für Workforce Management tätig. Das Unternehmen ist in mehr als 40 Ländern vertreten und gehört zu den Key Playern und Trendsettern in diesem Markt. Die Lösungen für nahezu alle Personalprozesse sind mittlerweile in acht Sprachen erhältlich. ATOSS-Lösungen sind exemplarisch in der Schweiz bei der Credit Suisse und bei Coop im Einsatz.

MICROSOFT ist weltweit einer der größten und bekanntesten Softwarehersteller und beschäftigt in der Schweiz ca. 620 Mitarbeitende. Microsoft ist nicht spezialisiert auf Personalinformationssysteme, in der von Microsoft angebotenen ERP-Lösung ist das Personalwesen aber ein integrierter Bestandteil. Referenzkunden im HR-Bereich sind zum Beispiel die deutschen Unternehmen Würth oder New Yorker.

ORACLE gehört ebenfalls zu den führenden Softwareherstellern weltweit. Mit seinen 420.000 Kunden in mehr als 145 Ländern bietet das Unternehmen eine umfassende Palette an Softwarelösungen an. Weltweit beschäftigt das Unternehmen mehr als 130.000 Mitarbeiter. Auch ORACLE deckt mit seinem HR-Softwareangebot nahezu alle Personalprozesse umfassend ab. Bekannte Schweizer Referenzkunden sind die Baloise Group oder das CERN.

Das im Jahre 2000 entstandene deutsche Unternehmen REXX SYSTEMS ist mittlerweile in über 20 Ländern vertreten und bietet ausschließlich HR-Lösungen an. 700 Kunden weltweit nutzen die einfach zu bedienende und hochskalierbare mehrsprachig verfügbare HR-Software. Schweizer Kunden sind u. a. die Helvetia und der Flughafen Zürich. 
Das von ehemaligen IBM-Mitarbeitern gegründete deutsche Unternehmen SAP sammelt seit mehr als 40 Jahren Erfahrungen im Bereich der Unternehmenssoftware. Mit über 310.000 Kunden in mehr als 190 Ländern ist die SAP im Bereich Unternehmenssoftware der umsatzstärkste Anbieter von Software und Softwareservices. Mittlerweile beschäftigt die SAP weltweit mehr als 78.000 Mitarbeitenden in 130 Ländern. Die HR-Lösungen sind modular und prozessorientiert aufgebaut. Vor allem Schweizer Großunternehmen wie SwissRe, Schweizerische Post, ABB oder die SBB setzen SAP-Systeme ein.

Als potenzielle Nachfrager von Digitalisierungsangeboten im Bereich HRM kommen alle Unternehmen, Organisationen und Institutionen infrage, die über ein kritisches Volumen an Personal(-bedarfen) verfügen. Da in der Literatur ein HRM-Digitalisierungsnachfragepotenzial eher unspezifisch thematisiert wird, kann ein auf die Digitalisierung generell abhebende Untersuchung zum Entwicklungsstand der Digitalisierung in der Schweiz, das Projekt digital.swiss, als Indikationserhebung für die weitere Entwicklung auch im HRM herangezogen werden. Die Studie leitet eine gesamtschweizerische Kennzahl ab, die den Fortschritt der Digitalisierung in der Schweiz vor dem Hintergrund des derzeit technisch Möglichen aufzeigt. Sie kommt zum Schluss, dass die Schweiz etwa die Hälfte des Wegs $(51 \%)$ hin zu einer digitalisierten Gesellschaft gegangen ist (ICT Switzerland 2016). Das höchste Entwicklungspotenzial weist das Thema „Industrie 4.0“ (Digitalisierungsscore von $18 \%$ ) auf. Am weitesten ist der Entwicklungsstand der Schweizer Basisinfrastrukturen mit einem Wert von $95 \%$. Der HR-seitig besonders interessante Wert für den Themenkreis „Fachkräfte \& Bildung“ liegt mit $56 \%$ leicht über dem Durchschnittswert. Das Nachfragepotenzial für Digitalisierungslösungen ist also auch hier noch sehr groß. Weitere branchenbezogene Untersuchungen von Ernst \& Young für die Bankenbranche und KMU (Computerworld 2016a, b) oder die breitere Studie von Accenture (2015) sehen ebenfalls noch erhebliches Wachstumspotenzial für Digitalisierungslösungen in der Schweiz, die derzeit vor allem im Kundenkontext zum Einsatz kommen. Generell am weitesten entwickelt scheinen Unternehmen im IT-, Kommunikations- und Medienbereich sowie die Banken.

\subsubsection{Problemfelder der Digitalisierung im Personalbereich}

Die Einführung von Digitalisierungsanwendungen im HR-Bereich ist nicht nur mit Vorteilen hinsichtlich der Prozesseffizienz und personalwirtschaftlicher Entscheidungen verbunden, sondern birgt auch eine Reihe potenzieller Problemfelder und Risiken.

Die Digitalisierung und die mit ihr verbundenen Möglichkeiten können für HR-Mitarbeitende Überforderungs- und Existenzängste nach sich ziehen, da insbesondere im administrativen Bereich erhebliche Einsparungen im Sinne einer besseren Wertschöpfung zu erwarten sind, die auch mit Personalreduzierungen einhergehen können, sofern die freiwerdenden Ressourcen nicht anderweitig eingesetzt werden. Positiv betrachtet heißt dies, dass Personalmitarbeitende zunehmend von Routinetätigkeiten entlastet und für anspruchsvollere und stärker kundenorientierte HR-Aufgaben frei werden. 
Eine wichtige Voraussetzung, um potenzielle Widerstände einer Digitalisierung des Personalwesens zu begegnen ist die Qualifizierung der betroffenen Personalmitarbeitenden. Das IT-/Computer-Basiswissen ist schweizweit generell bei den Berufstätigen noch unzureichend ausgeprägt, auch wenn dies individuell oft nicht so wahrgenommen wird. So überschätzen derzeit ca. $78 \%$ der Schweizer Bevölkerung ihre Computerkenntnisse teils erheblich. Dies ergab ein jüngst durchgeführter umfassender Wahrnehmungs- und Ist-Abgleich der ECDL Switzerland AG (2015). Eine fundierte Qualifizierung der HR-Mitarbeitenden im Umgang mit neuen Digitalisierungstools ist auch vor diesem Hintergrund zwingend. Gleiches gilt auch für benötigte Kompetenzen zum digitalen Wandel, die über die technische Basisqualifizierung der Personalmitarbeitenden hinausgehen. Eine neuere globale Befragung von 81 Unternehmen (Hoberg et al. 2015) hat ergeben, dass insbesondere grundlegende digitale Kompetenzen in den Bereichen Digitale Sicherheit, Business Change Management und Business Networks für eine erfolgreiche digitale Transformation in Unternehmen von Bedeutung sind. Dies sollte generell auch bei Neurekrutierungen außerhalb des Personalbereichs berücksichtigt werden.

Die Anwendung von HR-Digitalisierungslösungen birgt das Risiko, dass digitalisierte Prozessabwicklungen und Entscheidungsfundierungen zunehmend nicht mehr kritisch hinterfragt werden. Cachelin (2016b) spricht in dem Zusammenhang von sogenannten „Filter Bubbles“, wenn zum Beispiel eine Big-Data-basierte Personalvorauswahl dazu führt, dass nur noch gleichbleibende Mitarbeitendentypen rekrutiert werden, was die Innovationskraft von Unternehmen hemmen kann.

Die durch die Digitalisierung geförderte jederzeitige Datenverfügbarkeit geht auch mit einem wachsenden Risiko von Cyberattacken einher (Cachelin 2016b). Sensible Personaldaten sind hier in besonderem Maße gefährdet und zwingend bestmöglich vor Missbrauchszugriffen zu schützen. Eine aktuelle deutsche Unternehmensstudie zu IT-Sicherheit und Datenschutz (Schwartz und Muhle 2016) hat ergeben, dass für KMU insbesondere der Schutz von Software und Backup-Lösungen höchste Priorität haben und dass die Sicherheitsmaßnahmen bei allen Unternehmen umso größer ausfallen, je höher der technische Stand und der Vernetzungsgrad sind. Kaum ausgeprägt hingegen sind Maßnahmen im Bereich der Mitarbeitendenqualifizierung für Verhaltensänderungen und die Berücksichtigung von Sicherheitsaspekten bei der Rekrutierung neuer Mitarbeitender. Beides sind Bereiche, in denen die Personalabteilungen einen eigenen Beitrag für einen verbesserten Datenschutz leisten können. Ein besonders wichtiger Punkt bei der Diskussion des Datenschutzes in Verbindung mit einer Digitalisierung des HRM sind Cloud-Anwendungen, bei denen es vor allem auch darum geht, dass Informationen zu Logins, Datenbanken und weiteren sensiblen Informationen, die über das Internet zugänglich gemacht werden, nicht durch den Zugriff über ungesicherte Notebooks oder Mobilgeräte gefährdet werden. Darüber hinaus sind die Datenschutzmaßnahmen der Cloud-Anbieter durchaus nicht durchgängig zufriedenstellend (Cloudcomputing-insider 2016). Bei der Einführung von HRM-Cloud-Lösungen hat dieser Aspekt eine sehr hohe Relevanz. 


\subsubsection{Reifegradmodell}

In Anlehnung an Berghaus et al. (2015, S. 10 ff.) kann ein auf den Digitalisierungskontext des HRM abhebendes Reifegradmodell abgeleitet werden, das zur Systematisierung der eigenen empirischen Erhebung herangezogen wurde. Der St. Galler-Business-Engineering-Framework-Ansatz mit den Ebenen „Strategie“, ,Prozesse/ Organisation“ und „Technologie“ wird um die gleichsam relevanten HRM-Digitalisierungsbezugsebenen „Aktuelle und potenzielle Mitarbeitende“ (Kundenperspektive), „Kultur und Führung“ (Werteperspektive) sowie „HR-Services“ (Leistungsperspektive) erweitert und explizit in den HRM-Kontext gestellt. Ferner wird die Technologieebene durch die vier identifizierten technologischen HRM-Hauptentwicklungsfelder „Social Media“, „Data Mining“, „Cloud-Lösungen“ und „Mobility“ weiter ausdifferenziert, sodass das Modell aus neun Bewertungsfeldern mit unmittelbarem HRM-Bezug besteht (vgl. dazu Abb. 8.2). Auf die Bewertungsfelder werden fünf definierte Reifegradstufen angewendet, die für eine Gesamteinschätzung des Entwicklungsstands relevant sind. Anhand des Reifegradmodells soll somit differenziert und summarisch die Frage beantwortbar sein, wie der derzeitige Stand der HRM-Digitalisierung in der Schweiz zu bewerten ist.

Die fünf Reifegradstufen werden auf Basis einer Ist-Erhebung der Ausprägungen der Bewertungsfelder für ein Unternehmen ermittelt. Eine inhaltliche Präzisierung der Reifegradstufen findet sich in Tab. 8.2.

Die Charakterisierung der im HRM-Kontext zugrunde liegenden neun Untersuchungsdimensionen des Reifegradmodells erfolgt unter Bezugnahme auf eine Optimalausprägung eines digitalisierten Unternehmens (Digital Company) gemäß der definierten Reifegrade in Tab. 8.3.

Abb. 8.2 Reifegradmodell zur Digitalisierung des HRM in der Schweiz. (Eigene Darstellung in Anlehnung an Berghaus et al. 2015, S. 14)

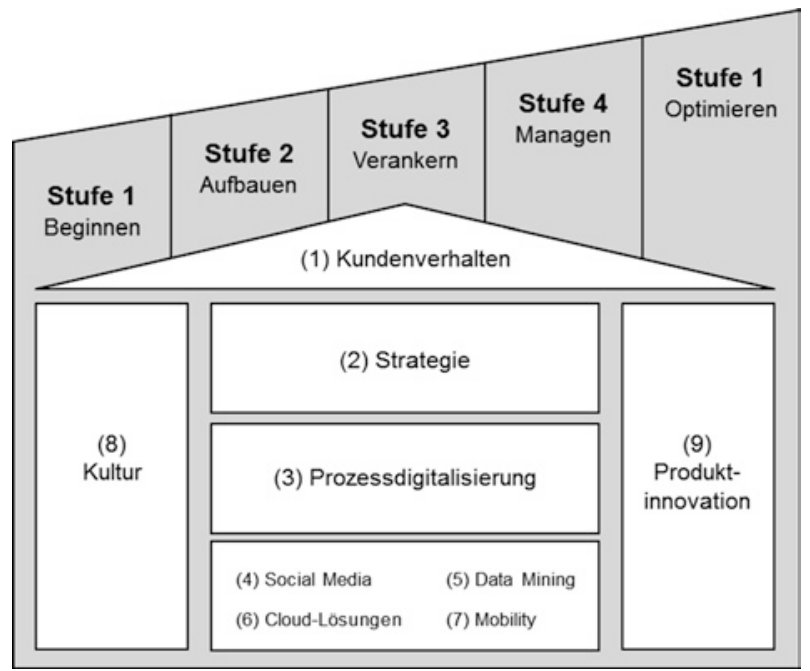


Tab. 8.2 Reifegrade zur Kennzeichnung des Digitalisierungsstands. (Quelle: Eigene Darstellung)

\begin{tabular}{l|l}
\hline Stufe & Beschreibung \\
\hline (1) Beginnen & $\begin{array}{l}\text { Prozesse sind noch nicht digitalisiert. Der Handlungsbedarf wird jedoch erkannt } \\
\text { und erste Ansätze für eine digitale Transformation sind erkennbar }\end{array}$ \\
\hline (2) Aufbauen & $\begin{array}{l}\text { Digitale Projekte werden priorisiert und vorangetrieben. Digitale Kompetenzen } \\
\text { sind bei der Rekrutierung von Mitarbeitenden eine wichtige Selektionsbasis }\end{array}$ \\
\hline (3) Verankern & $\begin{array}{l}\text { Das Thema „Digitalisierung“ ist auf strategischer Ebene verankert. Es wird als } \\
\text { Change-Projekt kontinuierlich und systematisch verfolgt. Digitale Schlüssel- } \\
\text { technologien für das Unternehmen sind definiert. Mobile Kanäle sind in allen } \\
\text { Leistungsfeldern integriert }\end{array}$ \\
\hline (4) Managen & $\begin{array}{l}\text { Neue digitale Technologien werden schnell aufgegriffen und überprüft. Neue } \\
\text { digitale Schlüsseltechnologien werden auf ihre strategische Bedeutung hin } \\
\text { analysiert. Die Automatisierung von Prozessen schreitet voran. Analytics-Tools } \\
\text { werden zur Optimierung eingesetzt und ausgebaut }\end{array}$ \\
\hline (5) Optimieren & $\begin{array}{l}\text { Der Digitalisierungsprozess wird systematisch und fortlaufend anhand von } \\
\text { Steuerungsindikatoren optimiert. Der digitale Entwicklungsstand in den Kern- } \\
\text { prozessen ist auf Best-Practice-Niveau }\end{array}$ \\
\hline
\end{tabular}

\subsection{Empirische Erhebung - Digital HRM in der Schweiz}

Das entwickelte Reifegradmodell dient als systematischer Dreh- und Angelpunkt zur Bewertung des Digitalisierungsgrades im HRM von Schweizer Unternehmen. Nachfolgend werden die Zielsetzung der empirischen Untersuchung, die methodischen Parameter und ihre Ergebnisse vorgestellt und kritisch reflektiert.

\subsubsection{Zielsetzung und Methodik}

Durch die Befragung von HRM-Experten Schweizer Unternehmen, die digitale HRM-Anwendungen einsetzen oder anbieten, soll ein (nicht repräsentativer) qualitativer Eindruck vom aktuellen Entwicklungsstand und bedeutsamen Trends bei technischen Lösungen für das HRM und deren Einsatz und Konsequenzen in der Praxis gewonnen werden. Darüber hinaus sollen Chancen und Problemfelder einer Digitalisierung im HRM erkannt und gestaltungsbezogene Empfehlungen abgeleitet werden.

Im Rahmen des gewählten induktiven qualitativen Forschungszugangs soll das „Wie“ des Beziehungsgefüges sowie dessen innere Struktur aus der Perspektive der Untersuchungsobjekte geklärt werden (Kiefl und Lamnek 1984, S. 474). Für Schweizer Unternehmen gibt es bis dato nur geringe Erkenntnisse zur Qualität und zum Ausmaß der Digitalisierung im HRM-Bereich. Grundlegend für die Konzipierung des Gesamtsettings der Erhebung war die Strukturempfehlung für empirische sozialwissenschaftliche Forschungsprozesse nach Gläser und Laudel (2006, S. 32). Das Forschungsziel 
Tab. 8.3 HRM-Dimensionen des Reifegradmodells am Beispiel einer Digital Company. (Eigene Darstellung)

\begin{tabular}{|c|c|}
\hline Dimension & Beschreibung \\
\hline $\begin{array}{l}\text { (1) Kundenver- } \\
\text { halten }\end{array}$ & $\begin{array}{l}\text { Digital Natives orientieren und bewegen sich in der Arbeitswelt bevor- } \\
\text { zugt auf digitalen Kanälen. Das HRM einer Digital Company trägt dem } \\
\text { dadurch Rechnung, dass Informationen zu gegenwärtigen und zukünftigen } \\
\text { Beschäftigten (Kunden) fortlaufend aus verschiedenen digitalen Quellen } \\
\text { generiert und zur Entscheidungsoptimierung eingesetzt werden (Verhaltens- } \\
\text { prognosen). Die Kommunikation mit aktuellen und potenziellen Mit- } \\
\text { arbeitenden erfolgt nahtlos und konsistent über alle digitalen Kanäle (z. B. } \\
\text { Self Service-Intranetplattformen). Gesellschaftliche und demografische } \\
\text { Veränderungen schlagen sich in einer kundenorientierten digitalen HR-Infra- } \\
\text { struktur nieder (hohe Usability und User Experience als Ziel) }\end{array}$ \\
\hline (2) Strategie & $\begin{array}{l}\text { In digitalisierten Unternehmen ist die Digitalisierung ein zentraler Bau- } \\
\text { stein von Geschäftsmodell und -strategie und auch der Personalstrategie. } \\
\text { Dies schlägt sich in optimierten Stellen- und Strukturlösungen für das } \\
\text { HRM und auf Mobilität, Flexibilität und Vernetzung ausgerichtete ganzheit- } \\
\text { liche Systemlösungen für HR-Manager und die Beschäftigen nieder (z. B. } \\
\text { Homeoffice-Angebot) }\end{array}$ \\
\hline $\begin{array}{l}\text { (3) Prozess- } \\
\text { digitalisierung }\end{array}$ & $\begin{array}{l}\text { In Digital Companies sind die internen HR-Prozesse durchgängig digitali- } \\
\text { siert, integriert und auf Prozesseffizienz und Kundenorientierung ausgerichtet } \\
\text { (z. B. Einsatz einer umfassenden HRM-Softwarelösung wie HCM Suite von } \\
\text { SAP) }\end{array}$ \\
\hline (4) Social Media & $\begin{array}{l}\text { Digitalisierte Unternehmen setzen in der Interaktion mit Menschen sehr } \\
\text { stark auf die sozialen Medien (YouTube, Xing, LinkedIn, Facebook etc.) } \\
\text { und tragen damit dem veränderten Kommunikationsverhalten relevanter } \\
\text { Zielgruppen Rechnung. Die breite professionelle Nutzung in verschiedenen } \\
\text { HR-Gestaltungsbereichen geht mit einer hohen Sensibilität in Bezug auf } \\
\text { mögliche Problembereiche einher (z. B. Erreichbarkeit, Qualität und Aktuali- } \\
\text { tät der Inhalte, Umgang mit Negativ- und Falschinformationen etc.) }\end{array}$ \\
\hline (5) Data Mining & $\begin{array}{l}\text { Digital Companies betreiben aktives und systematisches Data Mining und } \\
\text { verwandeln damit früher brachliegende Informationen über aktuelle und } \\
\text { potenzielle Mitarbeitende in wertgenerierendes Wissen. Der Einsatz von } \\
\text { Big Data-Applikationen und Business Intelligence-Lösungen für das HRM } \\
\text { ist selbstverständlich (z. B. Social Media-basierte Employer Branding-Ana- } \\
\text { lysen) }\end{array}$ \\
\hline $\begin{array}{l}\text { (6) Cloud- } \\
\text { Lösungen }\end{array}$ & $\begin{array}{l}\text { Cloud-Tools und -Systeme für den HR-Bereich (z. B. PeopleDoc) sind in } \\
\text { Digital Companies etabliert und stehen für innovative, flexible und kosten- } \\
\text { günstigere Anwendungsmöglichkeiten. Jeder Berechtigte kann von überall, } \\
\text { respektive von jedem internetfähigen Gerät auf HR-Daten zugreifen. }\end{array}$ \\
\hline (7) Mobility & $\begin{array}{l}\text { Mobile Geräte werden zunehmend auch zu universellen Arbeitsgeräten die } \\
\text { mit einer wachsenden Vernetzung aller Lebenssphären einhergehen. Digi- } \\
\text { talisierte Unternehmen tragen dem Rechnung, indem alle digitalen Lösun- } \\
\text { gen im HRM-Bereich auf eine sinnvolle mobile Nutzung hin geprüft und } \\
\text { entsprechend implementiert werden (z. B. Gehaltsabrechnung ist auf dem } \\
\text { Handy einsehbar). Responsive designte Applikationen sind Standard }\end{array}$ \\
\hline
\end{tabular}


Tab. 8.3 (Fortsetzung)

\begin{tabular}{l|l}
\hline Dimension & Beschreibung \\
\hline (8) Kultur & $\begin{array}{l}\text { Damit die Digitalisierung im HRM die intendierten Erfolgswirkungen } \\
\text { entfalten kann, ist eine große Offenheit der HR- und aller anderen Mit- } \\
\text { arbeitenden gegenüber neuen digitalen Technologien und der damit einher- } \\
\text { gehenden dynamischen Veränderungen erforderlich. Digitalisierung wird } \\
\text { in Digital Companies visionär, ,gelebt“ und ist als wichtiges Kulturelement } \\
\text { weitgehend etabliert (Digital Culture) }\end{array}$ \\
\hline $\begin{array}{l}\text { (9) Produkt- } \\
\text { innovationen }\end{array}$ & $\begin{array}{l}\text { Digitalisierte Unternehmen profilieren sich mit technologiebasierten Innova- } \\
\text { tionen bei HR-Dienstleistungen. Dies gilt auch und insbesondere in Bezug } \\
\text { auf die Anbieter von HR-Digitalisierungslösungen, die als Innovationstreiber } \\
\text { im HRM-Segment agieren (z. B. ADP, Atoss, Oracle, SAP etc.) }\end{array}$ \\
\hline
\end{tabular}

soll auf der Grundlage theoretisch definierter Vorabfestlegungen und einer resultierenden Samplebestimmung (Heister 2006) durch 17 leitfadengestützte Interviews von HR-Fachkräften und ein perspektivenweitendes und abrundendes Interview eines HR-Softwareexperten auf der Anbieterseite erreicht werden. Alle befragten Unternehmen sind in der Deutschschweiz ansässig. Bei der Auswahl der Unternehmen wurde wegen der hohen Zukunftsgerichtetheit der Themenstellung ein Fokus auf Großunternehmen gelegt, da diese aufgrund der Erkenntnisse der Voruntersuchung erheblich weiter bei der HRM-Digitalisierung fortgeschritten sind als das Gros der klein- und mittelständischen Unternehmen. Ausgangspunkt der Selektion geeigneter Partner war die Segmentas/ Handelszeitung-Top 500-Liste der größten Unternehmen der Schweiz mit mindestens 180 Mio. Franken Umsatz (Segmentas 2016). Bei der Auswahl der Probanden wurde auf ein möglichst breites Branchenspektrum geachtet. In Bezug auf die Anbieterseite konnte mit der SAP (Schweiz) AG einer der Marktführer für HRM-Digitalisierungslösungen gewonnen werden. Die Aussagen dieses Interviewpartners dienen primär der Validierung und Perspektivenweitung bei der Analyse der unternehmensbezogenen Aussagen auf der Nachfragerseite. Die teilnehmenden Unternehmen sind, mit Ausnahme von drei anonymisierten Großunternehmen, Tab. 8.4 entnehmbar. Für die Nachfrager- und Anbieterseite wurde auf Basis der Struktur des Reifegradmodells jeweils ein bewusst sehr offen gehaltener Fragebogen konzipiert und vorgängig getestet. Die Experteninterviews wurden im Zeitraum Juli bis November 2016 durchgeführt. Die aufgezeichneten Interviews wurden anschließend sinngemäß transkribiert, strukturell codiert und mit Microsoft Excel ausgewertet.

\subsubsection{Ergebnisse}

Nachfolgend werden die wichtigsten Ergebnisse der Erhebung in Anlehnung an die zugrunde liegende Struktur und Nummerierung des Reifegradanalysemodells dargelegt. 
Tab. 8.4 Befragte Schweizer Großunternehmen. (Eigene Darstellung)

\begin{tabular}{|c|c|c|}
\hline Unternehmen & Branche & Experte \\
\hline BKW AG & Energieversorgung/-verteilung & $\begin{array}{l}\text { Leitungsperson } \\
\text { HR }\end{array}$ \\
\hline BLS AG & Bahnen/Öffentlicher Verkehr & $\begin{array}{l}\text { HR-Expertin und } \\
\text { HR-Business } \\
\text { Partner }\end{array}$ \\
\hline Bystronic Laser AG & Maschinenindustrie & $\begin{array}{l}\text { Leitungsperson } \\
\text { HR }\end{array}$ \\
\hline Calida AG & Bekleidungsindustrie & $\begin{array}{l}\text { Zwei Leitungs- } \\
\text { personen HR }\end{array}$ \\
\hline Frutiger AG & Bau & $\begin{array}{l}\text { Leitungsperson } \\
\text { HR }\end{array}$ \\
\hline Genossenschaft Migros Aare & Detailhandel & $\begin{array}{l}\text { Leitungsperson } \\
\text { HR }\end{array}$ \\
\hline SAP (Schweiz) AG ${ }^{a}$ & Computer/Informatik & $\begin{array}{l}\text { Anonym (mit } \\
\text { HRM-Markt- } \\
\text { kenntnis) }\end{array}$ \\
\hline Schweizerische Mobiliar Holding AG & Versicherungen und Vorsorge & $\begin{array}{l}\text { Leitungsperson } \\
\text { HR }\end{array}$ \\
\hline Schweizerische Post AG & Dienstleistungen & $\begin{array}{l}\text { Leitungsperson } \\
\text { HR }\end{array}$ \\
\hline $\begin{array}{l}\text { Schweizerische Radio- und Fernseh- } \\
\text { gesellschaft (SRG SSR) }\end{array}$ & Mediendienstleistungen & $\begin{array}{l}\text { Leitungsperson } \\
\text { HR }\end{array}$ \\
\hline Schweizerische Bundesbahnen (SBB) & Bahnen/Öffentlicher Verkehr & HR-Experte \\
\hline Swisscom AG & Telekommunikation & $\begin{array}{l}\text { Leitungsperson } \\
\text { HR }\end{array}$ \\
\hline Valiant Bank AG & Banken & $\begin{array}{l}\text { Leitungsperson } \\
\text { HR }\end{array}$ \\
\hline Ypsomed AG & Medizinaltechnik & $\begin{array}{l}\text { Leitungsperson } \\
\text { HR }\end{array}$ \\
\hline $\begin{array}{l}\text { Unternehmen Automobil- und Motor- } \\
\text { fahrzeuggewerbe }\end{array}$ & $\begin{array}{l}\text { Automobil-/Motorfahrzeug- } \\
\text { gewerbe }\end{array}$ & $\begin{array}{l}\text { Leitungsperson } \\
\text { HR }\end{array}$ \\
\hline Bundesbehörde (im 3. Kreis) $^{\text {b }}$ & Öffentlicher Dienst & $\begin{array}{l}\text { Leitungsperson } \\
\text { HR }\end{array}$ \\
\hline Unternehmen Rüstungsgüterindustrie & Rüstungsgüterindustrie & $\begin{array}{l}\text { Leitungsperson } \\
\text { HR }\end{array}$ \\
\hline
\end{tabular}

${ }^{a}$ Anbieter von HRM-Digitalisierungslösungen (alle anderen Unternehmen sind Nachfrager)

${ }^{\mathrm{b}}$ Anstalt/Betrieb mit eigener Rechtspersönlichkeit, der zu $100 \%$ im Besitz des Bundes ist 


\section{(1) Kundenverhalten}

In Bezug auf die Analyse des Kundenverhaltens kann für nahezu alle untersuchten Unternehmen der Nachfragerseite festgestellt werden, dass die HR-Abteilungen bislang noch keine größeren Aktivitäten zur Erhebung der Bedürfnisse von aktuellen und potenziellen Mitarbeitenden hinsichtlich der Digitalisierung ergriffen haben. Digitale Innovationen im HRM werden eher technikgetrieben von außen und von internen Experten proaktiv auf der Grundlage von Annahmen zur Effizienzsteigerung angestoßen und gehen weniger von konkret erfragten Bedürfnislagen der Mitarbeitenden aus. Vereinzelt finden sich entsprechende Fragestellungen in Mitarbeitendenbefragungen wieder, in der Regel sind diese aber nicht sehr prominent lanciert. Losgelöst vom Digitalisierungsaspekt erfolgen allerdings in nahezu allen Unternehmen regelmäßige (Online-)Mitarbeitendenbefragungen und -gespräche. Ein kleiner Teil der Unternehmen verzichtet jedoch bewusst ganz auf Mitarbeitendenbefragungen oder ist im Zeitablauf von diesen aufgrund eines geringen Erkenntnisgewinns wieder weggekommen. Einen in technischer und inhaltlicher Hinsicht innovativen Ansatz verfolgt ein Unternehmen mittels eines webbasierten Tools, das es den Mitarbeitenden erlaubt über kurze Statements zu vordefinierten Themen Stimmungen wiederzugeben und an Entscheidungen mitwirken zu können.

In Bezug auf verhaltensbezogene Aspekte des demografischen Wandels im Kontext der Digitalisierung gibt es keine durchgängig einheitliche Einschätzung der Befragten. Der zumeist unterstellte Digitalisierungsvorsprung von jüngeren Mitarbeitenden wird von wenigen Experten sogar grundsätzlich infrage gestellt: Vereinzelt seien ältere Mitarbeitende deutlich technisch affiner eingestellt als jüngere. Das Gros der Befragten bestätigt aber grundsätzlich die Existenz größerer Wissens- und Erfahrungslücken zwischen älteren und jüngeren Generationen. Junge Mitarbeitende seien in der Regel wesentlich fundierter und vertrauter im Umgang mit neueren digitalen Entwicklungen und agieren oft als Treiber für digitale Innovationen in den Unternehmen. Ansatzpunkte für eine Angleichung der Digitalisierungsniveaus können Jung-Alt-Mentoring- oder Coaching-Modelle sein. Neben dem Alter kommen aber eine Reihe anderer Rahmensetzungen zum Tragen, die auf die Grundhaltung der Mitarbeitenden und deren Umgang mit der Digitalisierung einwirken wie etwa das Berufsfeld, die digitale Alltagsrelevanz oder die Dauer der Betriebszugehörigkeit und -prägung.

Die HR-Abteilungen der befragten Unternehmen haben den Digitalisierungstrend im Grundsatz zwar erkannt, bei einigen Unternehmen besteht aber offenbar noch ein spürbarer Nachholbedarf beim Einsatz von kundenorientierten digitalen HR-Lösungen. Insgesamt stehen die untersuchten Schweizer Unternehmen erst am Anfang, was die eingehende digitalisierungsbezogene Bedarfs- und Verhaltensanalyse von Mitarbeitenden angeht (Stufe 1 gemäß Reifegradmodell).

\section{(2) Strategie}

Die strategische Verankerung der Digitalisierung im HRM ist eine wichtige Voraussetzung für den Erfolg digitaler Transformationsprozesse. Für die meisten Befragten 
stellt die Digitalisierung einen unumgehbaren Megatrend dar (,vierte industrielle Revolution"), der die zukünftige Strategie und den nachhaltigen Erfolg der Organisation mitbestimmen wird. Gemäß der Einschätzung nahezu aller Befragter stehen die Unternehmen diesbezüglich aber erst am Anfang von sehr weitgehenden Veränderungen in der Arbeitswelt, aus denen neue Berufsbilder und Arbeitsformen hervorgehen werden. Erste größere strategische Digitalisierungsprojekte werden auf Ebene Gesamtunternehmen und im HR-Bereich auf den Weg gebracht. Ein Beispiel ist die Realisierung eines strategischen Omni-Channel-Projekts in einem Unternehmen, das zum Ziel hat, alle im Kontakt mit Bewerbenden und Mitarbeitenden eingesetzten Kanäle miteinander zu verbinden. Neben dem großen Potenzial für neue Geschäftsmodelle auf Gesamtunternehmensebene werden für den HR-Bereich vor allem Neugestaltungs- und Beschleunigungsoptionen für Personalprozesse als wichtig erachtet. Ein negativer Aspekt, der in dem Zusammenhang wiederholt genannt wird, ist die vermutete deutliche Einschränkung von künftigen Gestaltungsspielräumen für HR-Mitarbeitende.

Im strategischen Denken der HR-Probanden ist die Digitalisierung als strategisches Handlungsfeld durchgängig erkannt worden. Einzelne Unternehmen haben bereits HR-Projektexperten mit ausschließlichem Digitalisierungsfokus im Einsatz. Schon umgesetzte strategische Maßnahmen in Bezug auf die Digitalisierung des HRM sind allerdings derzeit eher noch die Ausnahme. Eine tendenziell vorsichtige, reaktive Grundhaltung der Probanden ist erkennbar. Auffallend ist allerdings, dass die großen staatsnahen Betriebe im Themengebiet in der Tendenz etwas progressiver unterwegs sind. Im Vergleich zur ersten analysierten Dimension kann für die Strategiedimension pauschal eine fortgeschrittenere Entwicklungsstufe attestiert werden. Die strategische Dimension der HRM-Digitalisierung befindet sich bei den untersuchten Großunternehmen im Aufbau (Stufe 2 gemäss Reifegradmodell).

\section{(3) Prozessdigitalisierung}

Bei der Analyse der dritten Dimension „Prozessdigitalisierung“ wird hinterfragt, in welchem Ausmaß interne HR-Prozesse durch die befragten Unternehmen bereits digitalisiert wurden.

Unabhängig von der Branche, der Unternehmensgröße oder der Rechtsform der untersuchten Unternehmen zeigt sich, dass das papierlose Büro im HR-Bereich seit einigen Jahren weitgehend realisiert ist. Eine Prozessdigitalisierung hat bei sämtlichen der befragten Unternehmungen mehrheitlich in denselben HR-Funktionsfeldern (Recruiting, Lohnabrechnung, Zeiterfassung) stattgefunden. Die Bereitschaft, Prozesse zu digitalisieren, steigt dabei mit der Höhe des Administrationsgrades des Prozesses an. Prozesse, welche einen großen administrativen Aufwand mit sich bringen, haben somit eine höhere Tendenz digitalisiert zu werden. Bei einer Detailbetrachtung der Umsetzungstiefe und -breite der Prozessdigitalisierung sind im Einzelvergleich signifikante Unterschiede erkennbar. Die Bandbreite reicht hier beispielhaft von eher traditionellen Standardumsetzungen im Bereich Online-Stellenausschreibungen und Bewerbermanagement über den Einsatz von Matching-Software im Recruiting und elektronische Personaldossiers bis 
hin zur toolbasierten Einbindung der Mitarbeitenden in das Employer Branding und hiermit verbundener Prozesse (Employer Advocacy). Eine Folge der relativ fortgeschrittenen Digitalisierung von HR-Standardprozessen ist die zunehmende Verlagerung von administrativen Arbeitsschritten auf die Kunden. Dies entspricht zumeist dem Bedürfnis der Mitarbeitenden nach selbstorganisierten HR-Services, kann aber auch negativ im Sinne einer Arbeitsverlagerung wahrgenommen werden. Wichtig für eine positive Wahrnehmung ist, dass die Prozessverbesserungen mit spürbaren Vereinfachungen einhergehen. So setzt ein Unternehmen beispielsweise bei der Erfassung von Arbeitszeiten bei externen Projekten Tablets ein, über die auch jederzeit die Lohnabrechnung abgerufen werden kann.

Dass die Prozessdigitalisierung das Personalwesen stark verändert, ist für alle Experten ein Faktum. In welchem Ausmaß dies der Fall ist, wird unterschiedlich eingeschätzt. Vielfach findet durch die Digitalisierung von HR-Prozessen lediglich eine Arbeitsveränderung und keine Arbeitseinsparung statt. Eingesparte Zeiten (z. B. durch das nicht mehr erforderliche Einscannen von Bewerbungsunterlagen) können nun durch HR-Mitarbeitende anders und produktiver genutzt werden. Darüber hinaus wird von einer erhöhten Dynamik, Vernetzung und Transparenz der Prozesse ausgegangen, was mit neuen, zumeist höheren Anforderungen an HR-Mitarbeitende einhergeht.

In Bezug auf die zeitliche Staffelung von Prozessdigitalisierungen wurden und werden offenbar administrative Prozesse wie die Arbeitsvertragsabwicklung, die Spesenabrechnung, die Lohnabrechnung und die Zeiterfassung tendenziell vor den restlichen HR-Prozessen digitalisiert. Kostenintensivere Tools mit nicht eindeutigem Mehrwert (z. B. ein Zeugnisgenerator) kommen eher nicht zum Einsatz. Nahezu alle HR-Bereiche sind bemüht, das Employer Branding und den Rekrutierungsprozess so digital wie möglich zu gestalten. Lediglich eines der untersuchten Unternehmen hat quasi sämtliche digitalisierbaren HR-Prozesse von der Rekrutierung bis zum Unternehmensaustritt von Mitarbeitenden auch digitalisiert (HR-Dokumentenplattform und Personendatenpflege, Zeiterfassungssystem mit Genehmigungsworkflows, elektronische Mitarbeiterbeurteilung, Aus- und Weiterbildungstool, Zeugniserstellungstool, Pensionskassenschnittstelle etc.). Vereinzelt werden die prozessbasierten HR-Services konzentriert in Shared Services-Centern bearbeitet.

Zusammenfassend kann festgehalten werden, dass die HR-Abteilungen der befragten Schweizer Unternehmen eine Reihe von Ansätzen der Prozessdigitalisierung bereits umgesetzt haben und hier Chancen für das HRM sehen. Der Entwicklungsstand ist bei einigen Unternehmen, die ganze Prozessketten umfassend abdecken, sehr hoch, bei anderen noch auf der Stufe von Standardprozessautomatisierungen verblieben und auf isolierte Prozessoptimierungen ausgerichtet. Im internationalen Vergleich zum Stand der Prozessdigitalisierung etwa mit nordamerikanischen Großunternehmen hinken die Schweizer Unternehmen zumeist noch hinterher. Über alle betrachteten Unternehmen hinweg kann summarisch von einer Prozessdigitalisierung im fortgeschritteneren Aufbau gesprochen werden (Stufe 2 gemäß Reifegradmodell). 


\section{(4) Social Media}

Die Analyse der vierten Dimension „Social Media“ befasst sich mit Art und Ausmaß der Einbindung von sozialen Netzwerken in das HRM der befragten Großunternehmen.

Die meisten HR-Experten sehen Social Media für die Zukunft als tragenden Baustein eines digitalisierten HRM, vor allem für die Rekrutierung und das Employer Branding. Im Umfeld der sozialen Medien geht es für die Unternehmen darum, sich positiv zu präsentieren und von der Konkurrenz abzuheben, aber auch um die Beschaffung von Informationen über ihre Zielgruppen am Arbeitsmarkt. Insbesondere die öffentlich zugänglichen Business-Plattformen Xing und LinkedIn werden von den HR-Experten als hierfür geeignet angesehen. Die HR-Social-Media-Auftritte werden zumeist aber nicht sehr aktiv betreut und befinden sich bei den meisten Unternehmen noch in der Auf- und Ausbauphase. Einzelne Unternehmen weichen auch hier positiv vom Gros des Samples $\mathrm{ab}$ und rekrutieren bereits intensiv, zum Teil via Active Sourcing, über Business-Plattformen. Private Netzwerke wie Facebook und Instagram werden HR-seitig bislang offenbar nur rudimentär von den Unternehmen genutzt und gepflegt. In Bezug auf die im Internet präsenten Bewertungsplattformen, wie zum Beispiel Kununu, gibt es kein einheitliches Bild. Die Bewertungen dieser Plattformen durch die HR-Experten divergieren stark. Einige Unternehmen agieren proaktiv andere reaktiv im Umgang mit den Plattformen und weitere Unternehmen ignorieren sie völlig, beziehungsweise sehen diese kritisch und scheuen den erforderlichen Zeitaufwand für eine Plattformbetreuung. Snapchat und Twitter, die von vielen Mitarbeitenden privat genutzt werden, werden bis anhin als Plattform für HR-Aktivitäten nur minimal oder gar nicht genutzt.

Alle Unternehmen sind sich darin einig, dass Social Media-Aktivitäten HR-seitig zunehmend bedeutsamer werden, und dass eine professionelle Nutzung von interaktiven Social-Media-Kanälen, neben der Notwendigkeit eines entsprechenden Know-hows, vor allem mit hohem zeitlichen Ressourceneinsatz verbunden ist. Aktuell befindet sich die Mehrheit der befragten Unternehmen noch in einer frühen Phase einer HR-Social Media-Erschließung. Integrative Gesamtlösungen mit breit vernetzten und anderen digitalen HR-Aktivitäten abgestimmten HR-Social Media-Aktivitäten sind derzeit bei den Unternehmen noch nicht oder nur ansatzweise im Einsatz. Die Social Media-Aktivitäten der untersuchten Unternehmen werden vor diesem Hintergrund summarisch noch dem Aufbaustadium zugeordnet (Stufe 2 gemäß Reifegradmodell).

\section{(5) Data Mining}

Nur etwa ein Fünftel der befragten Unternehmen investieren eher geringe finanzielle Mittel in den Auf- und Ausbau von Data Mining-Aktivitäten im HRM. Die Thematik wird als sehr populär betrachtet, konkrete Nutzungsoptionen sieht man aber eher in der Zukunft. Nur drei der interviewten Experten messen der Analyse von Big Data einen tiefen Stellenwert bei, alle anderen halten die Entwicklungen in diesem Bereich jedoch, insbesondere in Großunternehmen, für relevant und wichtig für das HRM (der Zukunft). Ein befragtes Unternehmen untersucht derzeit die Interaktionen und die Kommunikation von freiwillig mitwirkenden Mitarbeitenden, um Muster zu identifizieren, 
aus denen eventuelle mehrwertschaffende Maßnahmen abgeleitet werden können. Ein anderes Unternehmen setzt eine Absenzenmanagementanwendung ein, die bei Auffälligkeiten Führungskräfte automatisch per Mail informiert. Beide Beispiele stehen für eher rudimentäre Data Mining-Anwendungen mit Ausbaupotenzial in den befragten Unternehmen. Die im HR-Kontext besonders wichtige Anforderung der jederzeitigen Gewährleistung des Datenschutzes und der Einhaltung von gesetzlichen Vorgaben im Umgang mit Mitarbeitendendaten beim Einsatz von HR-Data Mining wird von allen Experten so betont. Die Gefahren, die mit dem Einsatz von HR-Data Mining verbunden sind, werden von nahezu allen Befragten gesehen. Neben der Gefahr des Datenmissbrauchs und von „Big Brother“-Ängsten in der Belegschaft sind dies vor allem auch Risiken der Fehlinterpretation aufgrund von veralteten oder qualitativ schlechten Daten oder die Entstehung von unproduktiven Datenfriedhöfen und der fachlichen Überforderung von HR-Mitarbeitenden, die in der Regel weder Mathematiker noch Statistiker sind. Etwa die Hälfte der Experten, vor allem der größeren Unternehmen, betont aber die Chancen für Verbesserungen von Entscheidungen im HRM durch die Analyse von HR-Big Data.

Erste HR Business Intelligence-Lösungen werden von etwas mehr als einem Drittel der untersuchten Unternehmen aktuell eingesetzt. Ein weiteres Fünftel prüft auf analytischer Ebene eine Einführung möglicher Anwendungen. Ein in diesem Themenfeld aktives Unternehmen führt beispielsweise momentan ein neues HR BI-Tool ein, das stark auf Visualisierung von Sachverhalten für die Linienführungskräfte abhebt und die Mitarbeitendenportfolios mit einer Fülle an Detailinformationen zu den einzelnen Mitarbeitenden jederzeit einsehbar macht. Ein weiteres Unternehmen setzt vermehrt auf die Vernetzung von Nicht-HR- mit den HR-Daten auf einer Plattform mit dem Hauptziel der Optimierung von Arbeitsabläufen. Die in der Literatur oft hervorgehobene zunehmende Relevanz von Prognoseoptionen und der Möglichkeit zur Trendidentifikation kann durch die Befragungsergebnisse nicht bestätigt werden. Abschließend kann für den Themenkreis HR Data Mining, respektive HR Big Data und HR Business Intelligence aggregiert ein Entwicklungsstand bestätigt werden, der erst am Anfang zu stehen scheint (Stufe 1 gemäß Reifegradmodell).

\section{(6) Cloud-Lösungen}

Die sechste Dimension des Reifegradmodells umfasst den Entwicklungsstand der Unternehmen in Bezug auf die Realisierung von HR-Cloud-Lösungen. Alle HR-Experten betonen die zunehmende Wichtigkeit, von überall und in verschiedensten Arbeitsprozessen auf die HR-Daten zugreifen zu können, um den gestiegenen Ansprüchen der Mitarbeitenden und Führungskräfte in Bezug auf Mobilität und Flexibilität genüge zu leisten, etwa wenn Tochtergesellschaften enger in das Unternehmensnetzwerk der Mutter eingebunden werden sollen. Darüber hinaus werden Cloud-Lösungen auch nicht unbeträchtliche Kostensenkungspotenziale zugewiesen, die relativ schnell und mit deutlicher Entlastung der unternehmensinternen kostenträchtigeren IT- und Supportstrukturen realisierbar sind. Von einigen Experten wird die in Verbindung mit den angeführten Eigenschaften verbundene hohe Einfachheit von Cloud-Lösungen ferner als große 
Chance zur Optimierung von HR-Prozessen durch Standardisierung gesehen. Nahezu alle Befragten bestätigen vor diesem Hintergrund eine hohe Wichtigkeit entsprechender Digitalisierungsoptionen. Die HR-Cloud gehört heute schon, und in Zukunft wohl noch in vermehrtem Umfang, zum Geschäftsalltag des Personalmanagements. Das Gros der untersuchten Unternehmen greift also bereits auf entsprechende Digitalisierungslösungen zurück. Diejenigen Unternehmen, die bis dato noch ohne Cloud-Einsatz agieren, sind in ihrem diesbezüglichen Entscheidungsraum eingeschränkt, zum Beispiel durch rechtliche Restriktionen bei öffentlichen Unternehmen (aktuell wird deshalb auf Bundesebene eine „Bundes-Cloud“ für öffentliche Institutionen diskutiert), aus Sicherheitsgründen in einem sensiblen Branchenumfeld (Hack-Gefahr) oder aufgrund anderweitiger technischer Lösungsansätze wie Corporate W-LAN. In funktionaler Hinsicht werden hauptsächlich Prozesse wie das Rekrutierungs- und Bewerbungsmanagement, die Lohnabwicklung, die Zeiterfassung und das HR Performance Management in die Cloud ausgelagert. Nur bei wenigen Unternehmungen werden allerdings sämtliche Prozesse in einer einheitlichen Cloud-Lösung integriert.

Die befragten Experten stehen in ihrem privaten Umfeld Cloud-Lösungen positiv gegenüber. Familienfotos, Videos, persönliche Daten etc. werden von ihnen in die Cloud ohne größere Bedenken hochgeladen. Eine gewisse Grundskepsis kann hingegen bei fast der Hälfte der HR-Manager festgestellt werden, wenn es um sensible Personaldaten eines Unternehmens geht. Von Gesetzes wegen herrschen in der Schweiz eindeutige Restriktionen, wie Personaldaten behandelt werden müssen, und welche Daten eine Unternehmung für ihre Zwecke analysieren darf. Solche sensiblen Daten dann noch in eine Cloud auszulagern, verbreitet, bei aller Technikaffinität, eine gewisse Unsicherheit bei den HR-Verantwortlichen. Alle Befragten sind sich einig, dass Datenschutz und Datensicherheit hier einen zentralen Stellenwert einnehmen. Dies wird auch von der Anbieterseite bestätigt, die diesem Kundenbedürfnis mit umfassenden Authentifizierungs-, Sicherheits- und Backup-Systemen nachkommt. Eine hundertprozentig sichere Lösung gibt es allerdings in der heutigen Zeit nicht mehr. Das gilt jedoch auch für die in der Regel noch stärker gefährdeten eigenen Nicht-Cloud-Systemlösungen der Unternehmen. In dem Zusammenhang spielt der Standort der Personaldaten-Server eine wichtige Rolle. Die meisten Unternehmen haben Regelungen festgelegt, in welchem Land die Daten physisch gespeichert werden dürfen. Die Schweiz wird hier als vertrauenswürdigstes Land genannt. Die USA stehen hingegen zunehmend in kritischer Diskussion.

Ein losgelöst von den technisch-strukturellen Aspekten von HR-Clouds resultierendes Gefahrenfeld für die Mitarbeitenden wird von einigen Experten für das Gesundheitsmanagement gesehen. Die mit den Clouds verbundene Zeit- und Ortsungebundenheit erhöht das Risiko einer unerwünschten zu hohen Überschneidung von Berufs- und Privatleben mit negativen Konsequenzen für die Work-Life-Balance von oftmals besonders leistungswilligen Mitarbeitenden (Erhöhung des Burn-out-Risikos).

Insgesamt kommen HR-Cloud-Lösungen aus Sicht der Befragten also eine hohe aktuelle und zukünftige Bedeutung zu. Das HR-Traktandum wird durchgängig als wichtig betrachtet, es besteht aber noch eine Reihe von Unsicherheiten bezüglich 
der Anwendung, vor allem in Bezug auf die Datensicherheit. Breite und Tiefe der HR-Prozessabdeckung in den Unternehmen ist aber offenbar noch signifikant ausbaubar. Auch fehlt es augenscheinlich noch an einer umfassenden strategischen Einbettung von Cloud-Ansätzen im HRM. Trotzdem kann angesichts der bereits starken Verbreitung und Implementierungsoffenheit von einer gewissen ,Verankerung“ von HR-Cloud-Lösungen in den untersuchten Schweizer Unternehmen gesprochen werden (Stufe 3 gemäß Reifegradmodell).

\section{(7) Mobility}

Die Grundhaltung der befragten Schweizer Unternehmen gegenüber Mobility-Ansätzen kann durchweg als positiv bezeichnet werden. Vor allem die betrachteten Unternehmen mit öffentlich-rechtlichem Hintergrund scheinen hier eine Vorreiterrolle einzunehmen. Fünf der untersuchten Firmen gehören zu den Unterzeichnern der „Work Smart Charta“, die flexible, ortsunabhängige Arbeitsformen fördern möchten, um motivierende Rahmenbedingungen für ihre Mitarbeitenden zu schaffen, den Arbeitsmarkt besser erschließen zu können und die zur Verfügung stehenden Ressourcen sowie die Infrastruktur ,smarter“ zu nutzen (Work Smart 2016). Mobility und flexible Arbeitsmodelle gehen allerdings nicht zwingend in allen Bereichen einher. Unternehmen mit einem hohen Anteil von „Blue Collar“-Mitarbeitenden sind hier deutlich eingeschränkter als etwa IT-Dienstleistungsunternehmen mit größtenteils hochqualifizierten Mitarbeitenden. Mobility kann in diesem Kontext auch mit der Wahrnehmung einer Privilegierung der „WhiteCollar"-Mitarbeitenden einhergehen. Dies schafft neue, transparenzfördernde Regelungsbedarfe. Die in Verbindung mit den Cloud-Lösungen angeführte Gefahr von psychischen Problemen und Erschöpfungszuständen wird auch in Verbindung mit den MobilityLösungen gesehen. Darüber hinaus besteht hier aber auch ein größeres Missbrauchspotenzial in anderer Hinsicht, wenn Mobility dazu führt, dass die intendierte Flexibilität in Arbeitsvermeidung mündet.

Auf der Modell- und Instrumenteebene steht Mobility für die zunehmende Bedeutung von Gleit-, Jahresarbeitszeit und Teilzeitansätzen sowie Desk Sharing. Außerdem befassen sich die Unternehmen vermehrt mit Homeoffice-Konzepten. Die grundlegende Offenheit für die angeführten Modelle ist bei allen Unternehmen vorhanden, es bestehen jedoch deutliche Unterschiede, was die Regelungstiefe der konkreten Umsetzungen angeht. Die Bandbreite reicht hier aus Sicht der betroffenen Mitarbeitenden von ,sehr autonom“ bis zu ,sehr restriktiv“. Wie bei den Cloud-Lösungen fehlt auch in diesem Themenkreis oft eine personalstrategische Verankerung, was Interpretationsspielräume öffnet und in Verbindung mit fehlendem Effizienzreporting Spannungen im Arbeitsumfeld mobiler Mitarbeitender wahrscheinlicher macht.

Bezüglich der Umsetzung von standortbezogenen aktivitätsorientierten Arbeitsplatzkonzepten zeichnet sich für die befragten Unternehmen ein stark fragmentiertes Bild ab. So hat beispielsweise ein Dienstleistungsunternehmen an seinem Hauptstandort eine ,inspirierende Arbeitswelt“ für seine Mitarbeitenden geschaffen. Im Gegensatz zum Arbeiten an anderen Standorten kann dort die Arbeitszeit durch die Mitarbeitenden 
vollkommen eigenverantwortlich organisiert werden. Mit den flexiblen Arbeitsmodellen einher geht eine offenbar zunehmende Bedeutung des „Third-Place“-Konzepts (zum Beispiel flexible Nutzung von kurzfristig anmietbaren Büroräumlichkeiten an Bahnhöfen), das eine Mobilitätslücke zwischen Büro und Homeoffice schließt. Sofern es die architektonische Gestaltung der Räumlichkeiten zulässt, hat ein Teil der untersuchten Unternehmen das „Open Space“-Büro mit unpersönlichen Arbeitslandschaften, Meetingzonen und Wechselarbeitsplätzen eingeführt. Digitale Mobilität geht also tendenziell einher mit Flexibilisierung der Arbeitszeitmodelle und der Arbeitsräume. In Bezug auf die drei HR-Handlungsfelder kann aufgrund der Befragungsresultate von einer wechselseitigen Verstärkung ausgegangen werden.

Die Effizienz dezentraler, technikunterstützter flexibler Arbeitsmodelle hängt aus Sicht der Befragten offenbar stark von der Existenz einer Grundvertrauenskultur ab. Bedürfnisbezogene Mobilitätslösungen für Mitarbeitende wie Homeoffice, die sorgfältig auf die arbeitsorganisatorischen Erfordernisse abgestimmt sind, können aus Sicht der HR-Experten die Produktivität, Motivation und Mitarbeiterbindung spürbar erhöhen und auch zu einer Verbesserung der Soft Skills von Mitarbeitenden führen. Dies ist aber aus Sicht einiger Experten nicht für jedes Unternehmen und jede Mitarbeiterkategorie gleichermaßen realisierbar. Aus der Perspektive der HRM-Mitarbeitenden eröffnen sich außerdem neue Wege, einen intensiveren Kontakt zu den Linienführungskräften und -Mitarbeitenden zu pflegen.

Neben der Notwendigkeit einer „On-Off-Kompetenz“ mobiler Mitarbeitender besteht eine besondere Herausforderung neuer mobiler Konzepte in den veränderten sozialen Strukturen: Kommunikation und Information innerhalb eines Teams können „,verwässert“ werden. Es können sich unerwünscht homogene Gruppen bilden und Koordinationsbrüche entstehen. Vertrauen kann missbraucht werden und der Teamzusammenhalt schwinden. Dem muss durch organisatorische Maßnahmen wie fixe Arbeitstage mit kompletter Teampräsenz entgegengewirkt werden. Außerdem sind auch mobile Arbeitsplatzlösungen mit Sicherheitsproblemen verbunden, und es können Innovationsprozesse gehemmt werden. Die potenziellen Probleme werden von den befragten HR-Experten also durchaus erkannt.

Über alle untersuchten Unternehmen hinweg kann festgehalten werden, dass digitalisierungsbasierte Mobility-Lösungen in stark unterschiedlichem Ausmaß implementiert sind. Einige Unternehmen haben schon beachtliche Anstrengungen zur Mobilitätsförderung ihrer Mitarbeitenden unternommen, andere streben dies vertieft erst in der Zukunft an. Auch hier kann, analog zur Verbreitung von Cloud-Lösungen, den Unternehmen übergreifend eine gewissen „Verankerung“ von Mobility-Ansätzen attestiert werden (Stufe 3 gemäß Reifegradmodell).

\section{(8) Kultur}

Digitalisierung ist immer mit strukturellen und kulturellen Veränderungen in den Unternehmen verbunden. Dem trägt die achte Dimension des Reifegradmodells Rechnung, die die „softe“ Seite des digitalen Wandels HR-seitig beleuchtet. Seitens der befragten 
HR-Experten wird eine offene und dynamische Unternehmenskultur wiederholt als Voraussetzung für den digitalen Wandel genannt. Auf der Ebene von Subkulturen wird in Abhängigkeit vom Ausmaß der Betroffenheit von Mitarbeitenden durch den digitalen Wandel jedoch auf eine Differenzierungserfordernis hingewiesen. Die Digitalisierung wird als ein kulturrelevanter Aspekt unter einer Reihe anderer Unternehmensfaktoren wie die Technologieorientierung, Größe, Branche, regionale Verankerung oder die Firmenstruktur gesehen. Wie bei allen unternehmenskulturellen Phänomenen auch spielen das Verhalten des Topmanagements und der nachgelagerten Führungsebenen im Kontext der Digitalisierung eine wichtige Rolle. In allen befragten Unternehmen wird versucht, die kulturellen Voraussetzungen für den digitalen Wandel zu fördern, sei dies durch Schulungen und die Kommunikation bei Anlässen, inspirierende Arbeitswelten oder etwa durch den Einsatz von technischen Hilfsmitteln wie Tablets bei den Vorstellungs- und Mitarbeitendengesprächen oder Self Service-Angeboten für eine stärkere Einbindung der Mitarbeitenden in Personalprozesse. In Bezug auf wandelbegleitende Kulturmaßnahmen herrscht aber nachvollziehbar eine sehr große Heterogenität mit hoher Unternehmensspezifität vor. Ein wichtiger kultureller Stellhebel ist allerdings für alle Unternehmen die Rekrutierung von neuen Mitarbeitenden mit erwünschten digitalen Kompetenzprofilen und die Einbindung aller Mitarbeitenden bei großen Digitalisierungsprojekten. Vorhandene Mitarbeitende, die sich aus Angst oder mangelnden Fähigkeiten dem digitalen Wandel verschließen, geraten in Gefahr, nicht mehr wettbewerbs-, beziehungsweise arbeitsmarktfähig zu werden. Dem sollte das HRM durch die angeführten und gleichgerichteten Maßnahmen entgegenwirken.

In Bezug auf die HR-Mitarbeitenden schlagen sich neue digitalisierungsbedingte Stellenprofile (eHR-Experten) und die Auslagerung und Vereinfachung administrativer Prozesse in einigen Unternehmen in einem von der Literatur an sich schon lange postulierten, nun aber offenbar auch breiter real stattfindenden Paradigmenwechsel für das HRM-Personal nieder, der weg von administrativen Aufgaben hin zum qualifizierten Coaching und der Beratung der Linie durch die HR-Mitarbeitenden führt. Die wertschöpfende Ausrichtung der Personalabteilungen schreitet also weiter voran und macht in Bezug auf dazu stimmige Verhaltens- und Werteanpassungen auch vor den HR-Managern und -Mitarbeitenden nicht halt. Kompetenzen wie Abstraktions- und analytisches Denkvermögen werden damit einhergehend zunehmend wichtiger. Insgesamt gehen die Befragten eher von einer Verschiebung und einer qualitativen Veränderung von HR-Aufgaben als von einem kompletten Wegfallen von HR-Arbeit aus, auch wenn stellenbezogene Einsparpotenziale für das HRM noch gesehen werden.

In allen Unternehmen wird also die hohe Relevanz der Unternehmenskultur für eine erfolgreiche Digitalisierung erkannt und dem durch unterschiedliche HR-induzierte oder -unterstützte Maßnahmen entsprochen. Tendenziell scheint sich in den Unternehmen die Unternehmenskultur eher in der Folge als vorausgehend zur Digitalisierung zu verändern. Die Einbindung von wertrelevanten Aspekten der Digitalisierung wie Flexibilität, Mobilität oder die Wertschätzung digitaler Kompetenzen befindet sich summarisch über alle analysierten Unternehmen hinweg noch in der Aufbauphase (Stufe 2 gemäß Reifegradmodell). 


\section{(9) Produktinnovation}

Die Analyse der neunten Dimension des Reifegradmodells befasst sich mit der Entwicklung und Verwendung digitaler Produktinnovationen im HRM. Aus Anbietersicht scheinen für die Zukunft prozessbezogen vor allem in den Bereichen Employer Branding, Recruiting, Learning, HR Services und Talent-/Careermanagement noch Entwicklungsmöglichkeiten vorhanden zu sein. Diese Wahrnehmung deckt sich mit Eindrücken aufseiten der nachfragenden Unternehmen. Aus Sicht der HR-Experten kommen innovativen kundenorientierten und kostensparenden Self Service-Angeboten künftig eine noch wichtigere Rolle zu. Bei deren Einsatz gibt es aktuell in Bezug auf Breite und Intensität noch große Unterschiede zwischen den Unternehmen. Self Service-Lösungen kommen momentan vor allem in der Spesenabrechnung, Weiterbildung, bei Personalgewinnungsanträgen, der Lohnabrechnung, der Zeit- und Absenzenerfassung sowie der Personaladministration zum Tragen. Ausgebaute HR-Lösungen auf der Grundlage von künstlicher Intelligenz sind Stand heute noch in keinem der befragten Unternehmen im Einsatz, man verspricht sich diesbezüglich aber, bei aller Unsicherheit und Ungewissheit, für die Zukunft viel, zunächst wohl im Bereich der Rekrutierung oder beim Talentmanagement. HR Data Analytics-Ansätze werden zwar auf persönlicher Ebene von den HR-Experten zumeist eher zurückhaltend bewertet, für die Unternehmen werden aber in Zukunft eine weitere Intensivierung der Aktivitäten und damit verbundene Regelungsbedarfe (Persönlichkeitsrechte) gesehen. Als Gegenposition zum Big Data-Trend wird von einzelnen Experten für die Zukunft eine Entwicklung in Richtung „Small Data“ im Sinne von „Zurück zum Individuum“ und zum Faktor „Mensch“ in Unternehmen ausgemacht. Weitere potenzielle Trendbereiche einer Digitalisierung des HRM sehen einzelne Befragte im Bereich Virtual Reality, etwa über virtuelle Einblicke in Arbeitsabläufe oder ganze Arbeitswelten. Auch dem „Internet of Things“ wird ein gewisses Potenzial für das HRM beigemessen, wenngleich die Erwartungen hier noch wenig konkret sind.

Die neunte Dimension des Reifegradmodells setzt sowohl an der Angebots- als auch an der Nachfragerseite an. Auf der einen Seite gehen digitale Produktinnovationen in der Regel von den Anbietern neuer digitaler HRM-Systemlösungen aus, auf der anderen Seite muss für diese Angebote seitens der Nachfrager eine hinreichende Offenheit und Investitionsbereitschaft bestehen. Die Angebotssituation auf dem Schweizer Markt für HR-Digitalisierungslösungen kann durchaus als innovativ und zukunftsorientiert gekennzeichnet werden, unter anderem, da in dem Bereich über international agierende IT-Unternehmen ein hoher internationaler Diffusionsgrad besteht. Auch eine große, wenngleich nicht unkritische Offenheit und Aufgeschlossenheit der Personalmanager und der hinter ihnen stehenden Unternehmen für HR-Digitalisierungsinnovationen ist gegeben. Eine Reihe von innovativen Ansätzen kommt, auch unter Einbindung der Resultate der vorgängig betrachteten Dimensionen, zur Anwendung, wenngleich einige davon sehr unternehmensspezifisch ausfallen. Übergreifend kann in Bezug auf das Innovationspotenzial und die -bereitschaft der analysierten Schweizer Großunternehmen von einem ausgeprägten Gestaltungswillen ausgegangen werden, der über eine 
bloße Verankerung von Einzelinnovationen etwas hinausgeht: Man will Digitalisierungsinnovationen im HRM managen und tut dies auch (Stufe 4 gemäß Reifegradmodell).

Abschließend kann der Stand der Digitalisierung des HRM der betrachteten 16 Schweizer Großunternehmen über alle Dimensionen und alle Unternehmen hinweg als „fortgeschritten im Aufbau“ charakterisiert werden (Stufe 2 gemäß Reifegradmodell) (vgl. dazu Abb. 8.3). Eine Tendenz in Richtung „Verankerung“ der Digitalisierung ist summarisch betrachtet erkennbar. Einige Vorreiterunternehmen haben diesen fortgeschritteneren Reifegrad bereits erreicht oder sogar bereits überschritten.

Eine aggregierte Beantwortung der eingangs gestellten fünf Forschungsfragen erfolgt in der Übersicht in Tab. 8.5.

Die untersuchten Schweizer Unternehmen bewegen und verändern sich also digitalisierungsbedingt in allen identifizierten Trendbereichen, wenngleich beim Umsetzungsstand der Digitalisierung eine große Bandbreite ausgemacht werden konnte. Vor allem die untersuchten staatlichen oder staatsnahen Betriebe sind im HR-Digitalisierungsprozess schon recht weit vorangeschritten. Dies hängt vermutlich aber primär mit deren überdurchschnittlicher Größe, techniknahen Geschäftsfeldern und hohen administrativen Anteilen zusammen und weniger mit ihrer Rechtspersönlichkeit. Im administrativen Bereich steigt der Digitalisierungsgrad tendenziell bei hoher Verwaltungslastigkeit der HR-Prozesse. Der damit verbundene Aufgabenwandel in Personalabteilungen fördert die Entwicklung des HR-Bereichs in Richtung wertschaffende Service- und Beratungseinheit für die Linie. Wertschöpfung heißt dabei im Kontext Digitalisierung nicht nur Kostensenkung, sondern auch eine Effektivitätsund Effizienzsteigerung, eine bessere Wettbewerbspositionierung, Attraktivitätsverbesserungen und eine Erhöhung des Flexibilitätspotenzials im Unternehmen.

Abb. 8.3 Stand der HR-Digitalisierung in Schweizer Großunternehmen. (Eigene Darstellung)

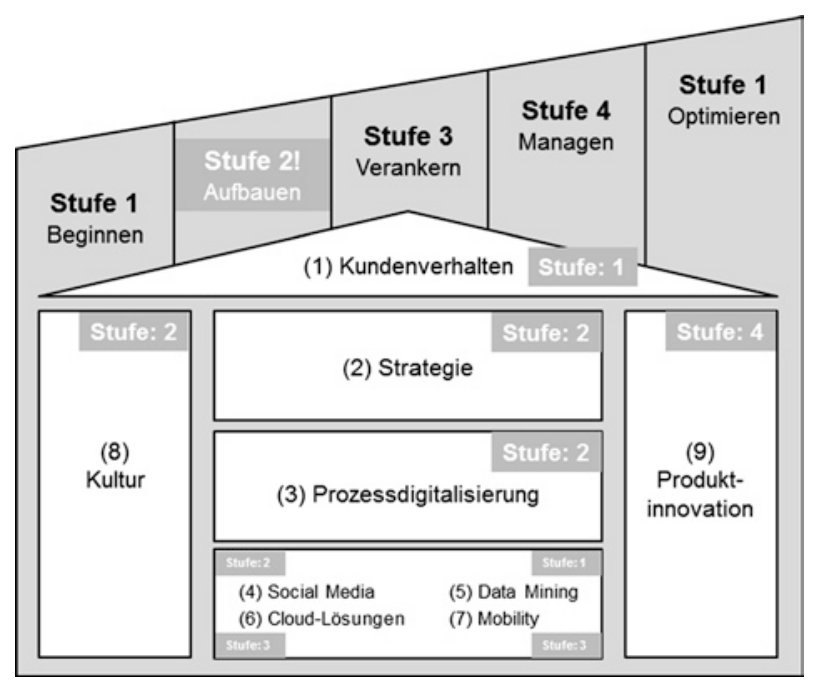


Tab. 8.5 Kernaussagen zu den fünf Forschungsfragen. (Eigene Darstellung)

\begin{tabular}{|c|c|}
\hline Forschungsfrage & Kernaussagen \\
\hline $\begin{array}{l}\text { Welche unter dem } \\
\text { Begriff der Digitalisie- } \\
\text { rung subsumierbaren } \\
\text { Trends sind für das } \\
\text { HRM weshalb von } \\
\text { Bedeutung? }\end{array}$ & 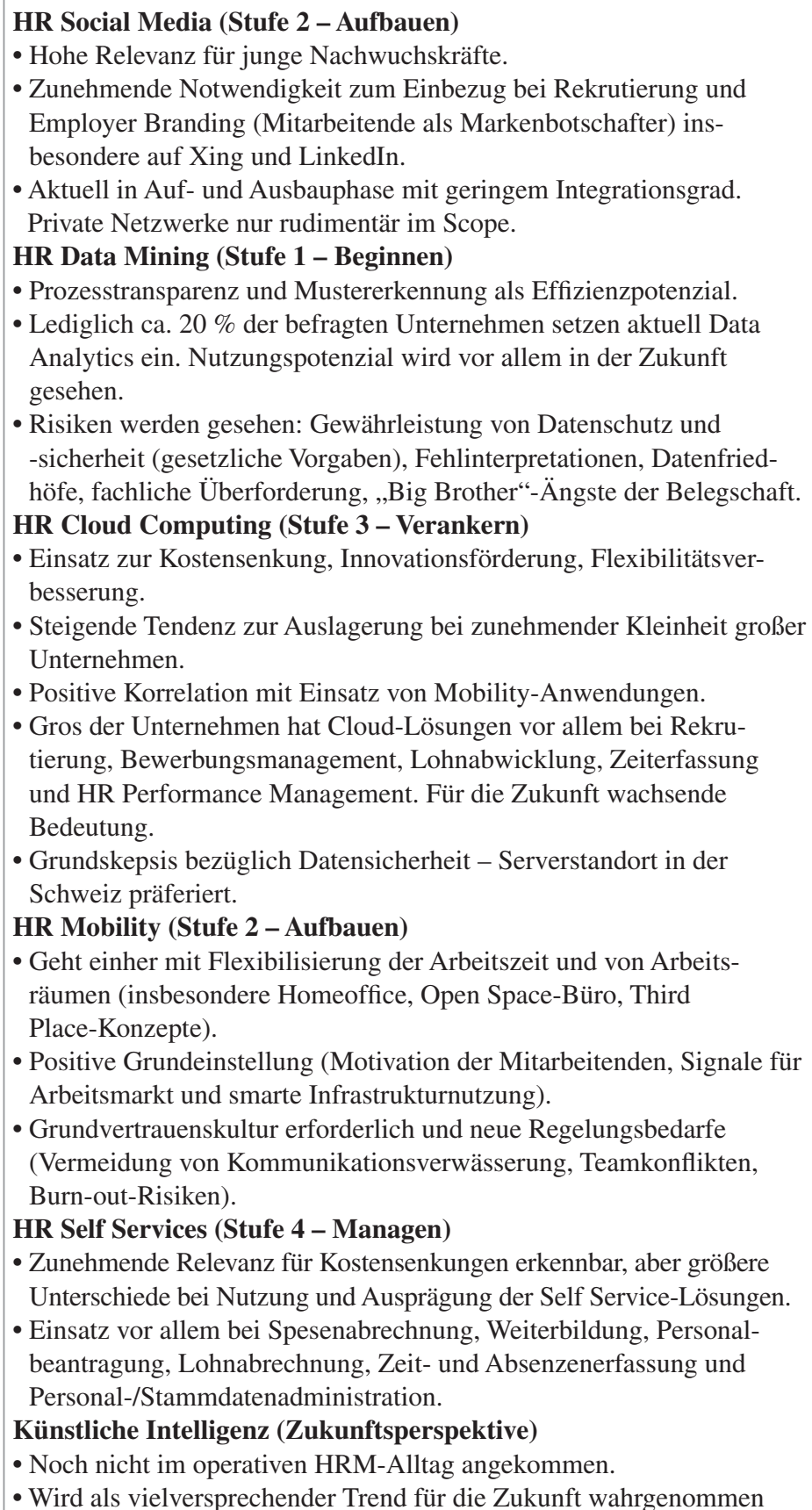 \\
\hline
\end{tabular}




\begin{tabular}{|c|c|}
\hline Forschungsfrage & Kernaussagen \\
\hline $\begin{array}{l}\text { Was sind in personaler } \\
\text { Hinsicht zentrale Chan- } \\
\text { cen und Problemfelder } \\
\text { der Digitalisierung? }\end{array}$ & $\begin{array}{l}\text { Chancen für das HRM } \\
\text { - Kostensenkungen und Qualitätsverbesserungen durch Prozess- } \\
\text { optimierung und Entscheidungsunterstützung. } \\
\text { - Unterstützt Wertschöpfung und Serviceorientierung im HRM: } \\
\text { Beratung statt Administration; strategische statt operative Personal- } \\
\text { arbeit. } \\
\text { Problemfelder für das HRM } \\
\text { - Datenschutz und Datensicherheit. } \\
\text { - Akzeptanz bei Mitarbeitenden (Self Services, „Big Brother“). } \\
\text { - Entgrenzung von Privat- und Berufswelt. } \\
\text { - Fachliche Überforderung von HR-Mitarbeitenden. } \\
\text { - Wachsende Abhängigkeit von Systemanbietern mit Folgekosten. }\end{array}$ \\
\hline $\begin{array}{l}\text { Welche Konsequenzen } \\
\text { und Gestaltungs- } \\
\text { empfehlungen lassen } \\
\text { sich aus den wich- } \\
\text { tigsten Trends für die } \\
\text { einzelnen Politikfelder } \\
\text { des HRM ableiten? }\end{array}$ & $\begin{array}{l}\text { - Trends beeinflussen alle Funktionsfelder, bzw. HR-Prozesse in mehr } \\
\text { oder minder großem Umfang. } \\
\text { - Positive Digitalisierungskultur und Neueinstellungen als wichtige } \\
\text { Stellhebel für die Zukunftsfähigkeit der Human Resources und des } \\
\text { HRM. } \\
\text { - Förderung von digitalen und von Beratungskompetenzen für HR-Mit- } \\
\text { arbeitende - Digital Change als Dauerzustand. } \\
\text { - Digital HR-Experte als neues Berufsprofil im HRM. } \\
\text { - Stärkere Zusammenarbeit zwischen IT- Bereichen und HRM zur } \\
\text { gemeinsamen Change-Bewältigung. } \\
\text { - Stärkere Bedarfsorientierung bei Digitalisierung im HR-Bereich (nicht } \\
\text { alles, was technisch möglich ist, ist auch gut für die „Kunden“ in der } \\
\text { Linie). }\end{array}$ \\
\hline $\begin{array}{l}\text { Wie sind einzelne } \\
\text { Digitalisierungstrends } \\
\text { aktuell im HRM } \\
\text { Schweizer Unter- } \\
\text { nehmen eingegangen? }\end{array}$ & $\begin{array}{l}\text { - Anstöße für neue HR-Digitalisierungslösungen kommen entweder } \\
\text { von internen Experten oder von externer Anbieterseite (Spezialisten- } \\
\text { lösungen mit eher gering konkretisierter Bedarfsorientierung in Bezug } \\
\text { auf Mitarbeitende und Führungskräfte als HR Service-Kunden). } \\
\text { - Kostensenkung, Arbeitgeberattraktivität und damit verbundene } \\
\text { Wettbewerbsfähigkeit am Arbeitsmarkt als kausale Haupttreiber der } \\
\text { Digitalisierung. }\end{array}$ \\
\hline $\begin{array}{l}\text { Wie, bzw. in welche } \\
\text { Richtung entwickelt } \\
\text { sich das HRM Schwei- } \\
\text { zer Unternehmen im } \\
\text { Kontext der Digitali- } \\
\text { sierung in den nächsten } \\
\text { Jahren weiter? }\end{array}$ & $\begin{array}{l}\text { - Wachstumstendenzen in allen identifizierten Trendbereichen. } \\
\text { Geschwindigkeit der Umwälzungen ist ungewiss, aber tendenziell } \\
\text { hoch. } \\
\text { - Weitere forcierte Fortführung von HR-Prozessautomatisierungen. } \\
\text { - HRM als wertschöpfungsgenerierendes internes Service-Center mit } \\
\text { hohem Digitalisierungsgrad. } \\
\text { - Digitalisierungsbedingte Profilveränderungen für HR-Mitarbeitende. }\end{array}$ \\
\hline
\end{tabular}




\subsection{Diskussion und Forschungsperspektiven}

Die theoretische und empirische Analyse der Digitalisierung im Kontext des HRM Schweizer Großunternehmen hat großteils Übereinstimmungen, aber auch einige Differenzen und Pointierungen hervorgebracht. So kann etwa der hohe Stellenwert und die teils enthusiastische Bewertung von HR Data Analytics in früheren Studien durch die eigene Untersuchung nicht bestätigt werden. Die Befragten sehen diesen Entwicklungstrend für die Zukunft zwar als sehr relevant an, erkennen aber durchaus auch kritische Aspekte dieses Themenkreises, die die weitere Entwicklung beeinträchtigen können. Eine hohe Konvergenz der theoretischen und der eigenen empirischen Perspektive konnte in Bezug auf die Notwendigkeit einer strategischen Verankerung der Digitalisierung festgestellt werden, wenn mit ihr die postulierten positiven Effektivitäts- und Effizienzeffekte realisiert werden sollen. In der Realität ist diese Verankerung aber zum gegenwärtigen Zeitpunkt eher noch eingeschränkt gegeben. Fehlende Digitalisierungsstrategien gehen mit Defiziten und Unsicherheiten bei der Implementierung von HR-Digitalisierungslösungen einher. Diesbezüglich sind sich die HR-Experten auf einer übergreifenden Argumentationsebene einig. Es mangelt bei einigen Unternehmen offenbar noch an einer ganzheitlichen Sicht auf die HR-Digitalisierung, die auch strukturelle und kulturelle Aspekte mit einbezieht (perspektivische Triade: Digital Strategy - Digital Structure - Digital Culture). Im Vergleich mit früheren Studien fällt auf, dass die Bedeutung der Social Media-Nutzung offenbar markant zugenommen hat (HR Social Media Multichannel Management). Prioritäre Nutzungsbereiche (Rekrutierung und Employer Branding) und relevante Plattformen (Xing, LinkedIn, Facebook) früherer Analysen konnten bestätigt werden. Die Plattform YouTube scheint aber vor dem Hintergrund der eigenen Erhebung in vorangehenden Untersuchungen hinsichtlich der untersuchten Firmengruppe überbewertet zu sein. In Ergänzung zu den in der Theorie vorgefundenen kritischen und unkritischen Beiträgen in Bezug auf die Verwässerung von Arbeitszeit und Freizeit kann für die befragten HR-Experten ein durchaus vorhandenes kritisches Grundbewusstsein attestiert werden, das gesundheitliche Überlegungen mit einbezieht. Die durch die Digitalisierung geschaffenen Möglichkeiten sollen demnach so genutzt werden, dass die Mitarbeitenden dies als motivationserhöhenden Zugewinn an Arbeits- und Lebensqualität empfinden und nicht als arbeitsbelasteten Zugriff auf die Privatsphäre. Flexibilität der Mitarbeitenden darf in langfristiger Sicht auch im Sinne der Unternehmen nicht zum Selbstausbeutungsmodell besonders engagierter Mitarbeitender verkommen. Der vielfach postulierte hohe Entwicklungsstand der Digitalisierung konnte insgesamt für den HR-Bereich nur eingeschränkt bestätigt werden. Hier ist nach wie vor auch bei Großunternehmen von einem hohen Entwicklungspotenzial auszugehen, das bei klein- und mittelständischen Unternehmen wohl noch um ein Vielfaches höher liegen dürfte. 
In Bezug auf die angewandte Forschungsmethodik ist auf eine Reihe potenziell kritischer Aspekte hinzuweisen. Das qualitative Forschungssetting mit einer relativ geringen Samplegröße und der Konzentration auf einen bestimmten (Deutsch-)Schweizer Unternehmenstypus schränkt die Repräsentativität und Generalisierbarkeit der Aussagen stark ein. Diese Defizite sind der Preis für eine größere inhaltliche Substanz und Breite der gemachten Aussagen im Sinne einer differenzierten aktuellen Momentaufnahme mit hohem deskriptivem Gehalt. Die methodisch fundierte Umsetzung der Interviewführung und -aufbereitung (Transkription und Codierung) mittels der Systematisierungsstruktur des entwickelten Reifegradmodells spricht ferner für eine gewisse Güte der analytischen Ergebnisse (Verfahrensdokumentation) (siehe hierzu und zur nachfolgenden Argumentationsführung auch Mayring 2002). Eine argumentative Interpretationsabsicherung fand durch einen kontinuierlichen Abgleich theoretischer und empirisch gewonnener Erkenntnisse und der empirischen Aussagen untereinander statt. Der Forschungsprozess erfolgte ferner durch Anlehnung an das Prozessmodell von Gläser und Laudel (2006, S. 32) weitgehend regelgeleitet. Die Nähe zum Forschungsgegenstand sollte durch die Befragung von ausgewählten Fachexperten vor Ort im unmittelbaren Arbeitsumfeld der Probanden und durch eine transparente Darlegung von Forschungsinhalt und Forschungszielen ihnen gegenüber (Forschung für die Betroffenen) hergestellt werden. Die Forschungsergebnisse wurden nach Abschluss der Analyse den teilnehmenden Unternehmen zugestellt, ohne dass grundlegende Korrekturen vorgenommen werden mussten (kommunikative Validierung). Die Kombination der gewählten Methodik mit einer breiteren quantitativen Erhebung könnte zur weiteren Fundierung der gemachten Aussagen sicher spürbar beitragen (Triangulation). Eine weitere Option zur qualitativen Verbesserung der Analyse wäre die Einbindung einer größeren Zahl von Unternehmen der Anbieterseite gewesen, die ursprünglich vorgesehen, aber beim gegebenen zeitlichen Rahmen nicht realisierbar war. Deshalb wurden die Anbieteraussagen nur zur Abrundung, Validierung und Interpretationsabsicherung hinsichtlich der befragten HR-Experten der Nachfragerseite verwendet.

Die Ergebnisse der vorliegenden empirischen Erhebung können als Basis für zukünftige weitere Untersuchungen im ausgewählten Themengebiet herangezogen werden. Angesichts der hohen Dynamik des Forschungsfelds und der hohen Praxisrelevanz wären tiefer gehendere qualitative und quantitative Analysen für die verschiedenen Funktionsfelder des HRM, für die Produktlösungen und -innovationen auf Anbieterseite, für umgesetzte Digital Change-Konzepte (,Wie“), für die quantitative Operationalisierung realisierter Einsparungen und Qualitätsverbesserungen oder für die Entwicklung integrativer Implementierungsmodelle (Digital HRM System Fit) wünschenswert. Auch in Bezug auf das zugrunde gelegte eingeschränkte Sample wäre eine Ausweitung des Befragungsscopes auf alle (Groß-)Unternehmen der Schweiz, inklusive Tessin und Romandie oder auf andere Unternehmensgrößen (KMU) von Interesse. 


\section{Literatur}

Accenture (Hrsg.). (2015). Digital Index Switzerland 2015. Biel: SAP AG intern (unveröffentlicht). Bankl, M. (2014). Lebensphasenorientierte Personalarbeit - Ein nachhaltiger Ansatz. In B. Rosenberger (Hrsg.), Modernes Personalmanagement: Strategisch-operativ-systemisch (S. 306-314). Wiesbaden: Gabler.

Beck, C. (2008). Personalmarketing 2.0: Vom Employer Branding zum Recruiting. Köln: Luchterhand.

Berghaus, S., Back, A., \& Kaltenrieder, B. (2015). Digital Transformation Report. St. Gallen: Institut für Wirtschaftsinformatik, Universität St. Gallen.

Botfrei Blog. (2012). Cloud-Computing - Oder was is teigentlich die Cloud? https://blog.botfrei. de/2012/12/cloud-computing-oder-was-ist-eigentlich-die-cloud/. Zugegriffen: 25. Mai 2016.

Cachelin, J. L. (2013a). Digitalisierung als Reifeprozess. Personalmagazin, 11(13), 51-53. https:// www.wissensfabrik.ch/digitalisierung-als-reifeprozess/. Zugegriffen: 25. Apr. 2016.

Cachelin, J. L. (2013b). HRM Trendstudie 2013. https://www.wissensfabrik.ch/hrmtrend2013/. Zugegriffen: 25. Mai 2016.

Cachelin, J. L. (2013c). Big Data Mining im HRM. https://www.wissensfabrik.ch/hr-big-data/. Zugegriffen: 25. Mai 2016.

Cachelin, J. L. (2014a). 5 Arbeitsfelder für das HR im digitalen Transformationsprozess. HR Today (3). https://www.wissensfabrik.ch/anleitungdigital/. Zugegriffen: 25. Apr. 2016.

Cachelin, J. L. (2014b). HR-Kompetenzen in einer digitalen Welt. Personal Manager, 2(2014), 22-24.

Cachelin, J. L. (2015). HRM Trendstudie. Risiken digitaler Arbeitswelten. https://www.wissensfabrik.ch/hrmtrend2014/. Zugegriffen: 25. Apr. 2016.

Cachelin, J. L. (2016b). Wissensfabrik. Risiken der digitalen Arbeitsumgebung. https://www. wissensfabrik.ch/digitalerisiken/\#software. Zugegriffen: 21. Mai 2016.

Cantin, F. (1999). Die innerbetriebliche Kommunikation verstehen und gestalten. Ein konstruktivistischer Ansatz und eine qualitative Analyse. Bern: Haupt.

Cloudcomputing-insider. (2016). Die 10 grössten Gefahren beim Einsatz von Cloud-Infrastrukturen. http://www.cloudcomputing-insider.de/die-10-groessten-gefahren-beim-einsatz-von-cloud-infrastrukturen-a-517146/. Zugegriffen: 28. Mai 2016.

Cole, T. (2015). Digitale Transformation. Warum die deutsche Wirtschaft gerade die digitale Zukunft verschläft und was jetzt getan werden muss!. München: Vahlen.

Computerworld. (2016a). Schweizer Banken: Digitalisierung erst am Anfang. http://www.computerworld.ch/news/it-branche/artikel/schweizer-banken-digitalisierung-erst-am-anfang-69399/. Zugegriffen: 9. Mai 2016.

Computerworld. (2016b). Schweizer KMU: Digitalisierung startet beim Kunden. http://www.computerworld.ch/marktanalysen/studien/artikel/schweizer-kmu-digitalisierung-startet-beim-kunden-69563/. Zugegriffen: 9. Mai 2016.

ECDL Switzerland AG (Hrsg.). (2015). Selbsteinschätzung und effektive PC-Kenntnisse der Schweizer Bevölkerung. Basel: ECDL Switzerland AG.

Eilers, S., Möckel, K., Rump, J., \& Schabel, F. (2016). HR-Report 2015/2016. Schwerpunt Unternehmenskultur. Bern: Hays AG.

Ernst \& Young (Hrsg.). (2016). Digitalisierung bei mittelständischen Unternehmen. Ergebnisse Kurzumfrage. http://www.ey.com/Publication/vwLUAssets/EY_Umfrage_Digitalisierung_mittelstaendische_Unternehmen_Schweiz/\$FILE/EY-Ergebnisse-Umfrage-Digitalisierung-2016.pdf. Zugegriffen: 25. Apr. 2016. 
Focus (2010). Was ist eigentlich Cloud Computing?. http://www.focus.de/digital/computer/kurzerklaert-was-ist-eigentlich-cloud-computing_aid_559544.html. Zugegriffen: 2. Juni 2016.

Gläser, J., \& Laudel, G. (2006). Experteninterviews und qualitative Inhaltsanalyse als Instrumente rekonstruierender Untersuchungen (2., durchgesehene Aufl.). Wiesbaden: VS Verlag.

Gora, W. (2016). Personalarbeit im Wandel: Digitalisierung als Chance, Personalpraxis24.de. http:// www.personalpraxis24.de/aktuelles/thema-der-woche/archiv-themen-der-woche/personalarbeitim-wandel-digitalisierung-als-chance/. Zugegriffen: 25. Apr. 2016.

Hackl, B., \& Gerpott, F. (2014). HR 2020 - Personalmanagement der Zukunft. München: Vahlen.

Haubrock, A. (2004). Personalmanagement. Stuttgart: Kohlhammer.

Heister, A. (2006). Qualitative Interviews - Ein Leitfaden zu Vorbereitung und Durchführung inklusive einiger theoretischer Anmerkungen. http://www.univie.ac.at/igl.geschichte/kaller-dietrich/WS\%2006-07/MEXEX_06/061102Durchf\%FChrung\%20von\%20Interviews.pdf. Zugegriffen: 30. Apr. 2016.

Heymann, B. (2009). Das Personalmanagement in mittelständischen Unternehmen. Hamburg: Igel.

Hoberg, P., Krcmar, H., Oswald, G., \& Welz, B. (2015). IDT Survey 2015 - Skills for digital transformation. Biel: SAP AG intern (unveröffentlicht).

Holtbrügge, D. (2015). Personalmanagement (6., neubearb. u. erw. Aufl.). Berlin: Springer.

HRM.ch (2013). HR-Software Report 2013 - die Ergebnisse. http://www.hrm.ch/fachartikel/ hr-software-report-2013-die-ergebnisse-10827. Zugegriffen: 27. Apr. 2016.

Huck-Sandhu, S. (Hrsg.). (2015). Interne Kommunikation im Wandel: Theoretische Konzepte und empirische Befunde. Heidelberg: Springer.

HWZ (Hrsg.). (2015). Über 50 Prozent der Unternehmen sind digitale Dinosaurier. http://www. fh-hwz.ch/de/news/news-detail/studie-digitale-transformation-2015.htm. Zugegriffen: 25. Apr. 2016.

ICT Switzerland (2016). Scorcard. http://digital.ictswitzerland.ch/. Zugegriffen: 9. Mai 2016.

Jäger, W., \& Petry, T. (2012). Entreprise 2.0 - Die digitale Revolution der Unternehmenskultur. Warum Personalmanager jetzt gefordert sind. Köln: Wolters Kluwer.

Karaus, S. (2003). Vergleich ausgewählter Anwendungsmöglichkeiten zur Nutzung des Elecetronic Human Resources Management in Unternehmen. Hamburg: Diplomica.

Kiefl, W., \& Lamnek, S. (1984). Qualitative Methoden in der Marktforschung. Planung und Analyse, 11(12), 474-480.

Kienbaum (Hrsg.). (2015). Ergebnisbericht HR-Trendstudie 2015. http://www.kienbaum.com/ Portaldata/1/Resources/downloads/brochures/Kienbaum_HR-Trendstudie_FINAL.pdf. Zugegriffen: 25. Apr. 2016.

Kolb, M. (2008). Personalmanagement. Grundlagen. Konzepte - Praxis. Wiesbaden: Gabler.

Kolb, M. (2010). Personalmanagement. Grundlagen und Praxis des Human Resources Management (2. Aufl.). Wiesbaden: Gabler.

Mayring, P. (2002). Qualitative Sozialforschung (5. Aufl.). Weinheim: Verlagsgruppe Beltz.

Nicolai, Ch. (2009). Personalmanagement (2. Aufl.). Stuttgart: Lucius \& Lucius.

Olsok, A., Kühn, T., Schulz, A., Bruhn, H., \& Kirch, J. (2016). Datenschutz im Personalmanagement. Gemeinschaftsstudie.. Berlin: Bitkom.

Pentaho (Hrsg.). (2012). BI4HR - Flexible Analyse und Reporting von Personaldaten. http://www. bi4hr.ch/download/BI4HR\%20Casestudy.pdf. Zugegriffen: 25. Mai 2016.

PwC (Hrsg.). (2013). HR und Social Media: Hier klickt der Zeitgeist. http://www.pwc.ch/user_ content/editor/files/publ_hrs/pwc_hr_und_social_media_d.pdf. Zugegriffen: 30. Mai 2016.

PwC (Hrsg.). (2014). Moving HR to the cloud? https://www.pwc.ch/user_content/editor/files/publ_ hrs/pwc_moving_hr_to_the_cloud_e.pdf. Zugegriffen: 25. Mai 2016.

Scherm, E., \& Süss, S. (Hrsg.). (2011). Personalmanagement. München: Vahlen. 
Scherm, E., \& Süss, S. (Hrsg.). (2013). Personalmanagement. München: Vahlen.

Schwarb, T., \& Vecchia, M. D. (2016). HRM - 4.0 Digitales Human Resource Management. Rheinfelden: BPX-Edition.

Schwartz M., \& Muhle, A. (2016). Chancen der Digitalisierung nutzen: Datenschutz und IT-Sicherheit gehören dazu. KfW Research Magazin, 117(8), Februar 2016. Frankfurt: KfW Research.

Segmentas (Hrsg.). (2016). TOP 500 Handelszeitung. Die grössten Unternehmen der Schweiz. http://www.segmentas.ch/top500. Zugegriffen: 13. Dez. 2016.

Selke, M. (2012). Personalmanagement. In C. Thielscher (Hrsg.), Medizinökonomie (S. 197-241). Wiesbaden: Gabler.

Spachmann, K., \& Huck-Sandhu, S. (2013). Interne Kommunikation - Stellenwert und Neuausrichtung. In Bentele, G., Piwinger, M., \& Schonborn, G. (Hrsg.), Loseblattwerk Kommunikationsmanagement (Beitrag 3.96). Neuwied: Luchterhand.

Spachmann, K., \& Huck-Sandhu, S. (2015). Zwischen Beteiligung und Dialog: Social Media in der internen Kommunikation. Ergebnisbericht zur Umfrage 2014. Pforzheim/Stuttgart: Hochschule Pforzheim/Universität Hohenheim. http://www.ik-trends.de/wp-content/ uploads/2015/07/IK2014_Social-Media.pdf. Zugegriffen: 27. Apr. 2016.

Stock-Homburg, R. (2008). Personalmanagement. Theorien, Konzepte, Instrumente. Wiesbaden: Gabler.

Tagesanzeiger. (2015). Hälfte der Firmen fehlen geeignete Arbeitskräfte. Tagesanzeiger Online, 17.08.2015. http://www.tagesanzeiger.ch/olympia2012/switzerland/haelfte-der-firmen-fehlengeeignetearbeitskraefte/story/16550916?track. Zugegriffen: 14. Apr. 2016.

Tetzel, T. (2016). Cloud Computing: SaaS, PaaS \& IaaS einfach erklärt. https://www.datenschutzbeauftragter-info.de/die-cloud-saas-paas-und-iaas-einfach-erklaert/. Zugegriffen: 25. Mai 2016.

Thom, N. (2001). Personalmanagement - Überblick und Entwicklungstendenzen. In N. Thom, \& R. J. Zaugg (Hrsg.), Excellence durch Personal- und Organisationskompetenz (S. 117-131). Bern: Haupt.

Wachter, T. (2015, August 18). Personalentwicklung: Das sind die Ziele. http://www.weka.ch/ themen/personal/personal-fuehrung-und-personalentwicklung/personalentwicklung/article/ personalentwicklung-das-sind-die-ziele/. Zugegriffen: 28 Apr. 2016.

Wagner AG (Hrsg.). (2016). BI/ Analytics. KMU Lösungen für BI und Analytics. http://www.wagner.ch/loesungen/bi-analytics.html. Zugegriffen: 25. Mai 2016.

Westerman, G., Bonnet, D., \& McAfee, A. (2014). Leading digital: Turning technology into business transformation. Harvard: Harvard Business Press.

Winkler, S. (2016). Was HR-Profis beschäftigt. HR-Today, 27.01.2016. https://www.hrtoday.ch/ article/was-hr-profis-beschäftigt. Zugegriffen: 26. Apr. 2016.

Work Smart. (2016). Was ist die Charta? http://work-smart-initiative.ch/de/überuns/charta-unterzeichnen/. Zugegriffen: 18. Dez. 2016.

Schellinger, Jochen (Prof. Dr./jochen.schellinger@bfh.ch) Studiengangsleiter Master of Science in Business Administration an der Berner Fachhochschule. Lehr- und Forschungstätigkeiten in den Bereichen Strategisches Management, Marketing und Personalmanagement. Davor 15 Jahre Praxistätigkeit bei der Deutschen Sparkassenorganisation, im Haniel-Konzern, an der Universität Tübingen sowie in der Daimler AG.

Goedermans, Marlies (M.Sc. BA/marlies.g@bluewin.ch) Abgeschlossenes konsekutives Masterstudium in Betriebsökonomie mit Vertiefung Geschäfts- und Unternehmensentwicklung an der Berner Fachhochschule. Derzeit als Finance Operations Specialist bei der SAP Schweiz beschäftigt. 
Kolb, Lars (M.Sc. BA/lars.kolb@gmx.ch) Abgeschlossenes konsekutives Masterstudium in Betriebsökonomie mit Vertiefung Geschäfts- und Unternehmensentwicklung an der Berner Fachhochschule. Derzeit als Product Manager mit Kaderfunktion bei Interdiscount - eine Division der Coop Genossenschaft - beschäftigt.

Sebai, Yassin (M.Sc. BA/yaessu@msn.com) Abgeschlossenes konsekutives Masterstudium in Betriebsökonomie mit Vertiefung Geschäfts- und Unternehmensentwicklung an der Berner Fachhochschule. Derzeit als Projektleiter Sponsoring \& Events bei der Schweizerischen Mobiliar Versicherungsgesellschaft beschäftigt.

Open Access Dieses Kapitel wird unter der Creative Commons Namensnennung 4.0 International Lizenz (http://creativecommons.org/licenses/by/4.0/deed.de) veröffentlicht, welche die Nutzung, Vervielfältigung, Bearbeitung, Verbreitung und Wiedergabe in jeglichem Medium und Format erlaubt, sofern Sie den/die ursprünglichen Autor(en) und die Quelle ordnungsgemäß nennen, einen Link zur Creative Commons Lizenz beifügen und angeben, ob Änderungen vorgenommen wurden.

Die in diesem Kapitel enthaltenen Bilder und sonstiges Drittmaterial unterliegen ebenfalls der genannten Creative Commons Lizenz, sofern sich aus der Abbildungslegende nichts anderes ergibt. Sofern das betreffende Material nicht unter der genannten Creative Commons Lizenz steht und die betreffende Handlung nicht nach gesetzlichen Vorschriften erlaubt ist, ist für die oben aufgeführten Weiterverwendungen des Materials die Einwilligung des jeweiligen Rechteinhabers einzuholen.

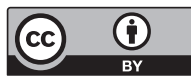

